\title{
DUALITIES FOR EQUATIONAL CLASSES OF BROUWERIAN ALGEBRAS AND HEYTING ALGEBRAS
}

\author{
BY
}

BRIAN A. DAVEY (1)

\begin{abstract}
This paper focuses on the equational class $S_{n}$ of Brouwerian algebras and the equational class $L_{n}$ of Heyting algebras generated by an $n$-element chain. Firstly, duality theories are developed for these classes. Next, the projectives in the dual categories are determined, and then, by applying the dualities, the injectives and absolute subretracts in $S_{n}$ and $L_{n}$ are characterized. Finally, free products and the finitely generated free algebras in $S_{n}$ and $L_{n}$ are described.
\end{abstract}

Recently there has been considerable interest in distributive pseudocomplemented lattices, Brouwerian algebras and Heyting algebras. In particular, activity has centered around the equational subclasses ([8], [11], [24], [35], [36]), and steps have been made towards the determination of the injectives, absolute subretracts, free products and free algebras in these classes $([1],[2],[3],[12],[19]$, $[20],[21],[27],[31],[32],[33],[34],[46],[47])$. In this work attention is focused upon the equational class $S_{n}$ of Brouwerian algebras and the equational class $\mathbf{L}_{n}$ of Heyting algebras generated by an $n$-element chain. Firstly, a duality theory is developed for each of these classes, the dual of an algebra being a Boolean space endowed with a continuous action of the endomorphism monoid of the $n$-element chain. Next, the projectives in the dual categories are determined, and then, by applying the dualities, the injectives and absolute subretracts in $\mathbf{S}_{n}$ and $\mathbf{L}_{n}$ are characterized. Finally, free products and the finitely generated free algebras in $S_{n}$ and $L_{n}$ are described.

1. The categories. Our standard references on category theory, universal algebra, and lattice theory are S. Mac Lane [37], G. Grätzer [17], and G. Grätzer [18] respectively; for our general topological requirements we refer to J. Dugundji [13] and for a discussion of Boolean a spaces we call on P. R. Halmos [23].

Received by the editors August 13, 1974.

AMS (MOS) subject classifications (1970). Primary 06A35, 18A40, 54H10; Secondary 02C05, 08A10, 08A25, 18C05, 54F05, 54G05.

Key words and phrases. Distributive lattice, Brouwerian algebra, Heyting algebra, relative Stone algebra, $L$-algebra, Boolean space, duality, projective, injective, weak injective, $a b$ solute subretract, free product, free algebra.

(1) Research supported by the Canada Council. 
A Brouwerian algebra $A$ is a (necessarily distributive) lattice in which, for all $a, b \in A$, there exists $a * b \in A$ such that $x \wedge a \leqslant b \Longleftrightarrow x \leqslant a * b$. Since $A$ necessarily has a unit, namely $a * a$, we regard Brouwerian algebras as universal algebras of type $\langle 2,2,2,0\rangle$ with operations $\langle\Lambda, V, *, 1\rangle$. A Heyting algebra is a Brouwerian algebra with zero, and so it is a universal algebra of type $\langle 2,2,2$, $0,0\rangle$ with operations $\langle\Lambda, \vee, *, 0,1\rangle$. The standard results on Brouwerian and Heyting algebras can be found in H. Rasiowa and R. Sikorski [43] where they are referred to as relatively pseudocomplemented lattices and pseudo-Boolean algebras respectively. In particular, recall that the classes of Brouwerian and Heyting algebras are equational and that the lattice of congruences on a Brouwerian or Heyting algebra is isomorphic to its lattice of filters. It follows immediately from the latter fact that each Brouwerian or Heyting algebra has a distributive congruence lattice, and that every equational class of Brouwerian or Heyting algebras has the congruence extension property (see Definition 4.1).

We denote the $n$-element chain, $0=c_{0}<c_{1}<\cdots<c_{n-2}<c_{n-1}=1$, as a Brouwerian algebra by $C_{n}^{1}$ and as a Heyting algebra by $C_{n}$. Note that in any chain $C$ the operation * of relative pseudocomplementation is determined as follows:

$$
a * b= \begin{cases}1 & \text { if } a \leqslant b \\ b & \text { if } a>b\end{cases}
$$

A relative Stone algebra is a Brouwerian algebra which satisfies the identity $(x * y) \vee(y * x)=1$. The equational class of all relative Stone algebras is denoted by $\mathbf{S}_{\omega}$ and, for $1 \leqslant n<\omega, S_{n}$ denotes the equational subclass generated by $C_{n}^{1}$. An L-algebra is a Heyting algebra satisfying $(x * y) \vee(y * x)=1$. The equation class of all $L$-algebras is denoted by $\mathbf{L}_{\omega}$ and, for $1 \leqslant n<\omega, \mathbf{L}_{n}$ denotes the equational subclass generated by $C_{n}$. It is well known ([2], [7], [8], [38]) that every interval in a relative Stone algebra is a Stone algebra; whence the name. (A bounded lattice $A$ in which the pseudocomplement $a^{*}=a * 0$ exists for all $a \in A$ is called pseudocomplemented. A Stone algebra is a distributive pseudocomplemented lattice satisfying the identity $x^{*} \vee x^{* *}=1$.) Relative Stone algebras date back to G. Grätzer and E. T. Schmidt [22] and $L$-algebras arise naturally in the study of intermediate logics ([26] , [27]). T. Hecht and T. Katrinák [24] have shown that the lattices of equational subclasses of $S_{\omega}$ and $L_{\omega}$ are given by the $(\omega+1)$-chains $S_{1} \subset S_{2} \subset \cdots \subset S_{\omega}$ and $L_{1} \subset L_{2} \subset \cdots \subset L_{\omega}$, and that $S_{n}$ and $L_{n}$ are characterized by the identity

$$
\left(x_{0} * x_{1}\right) \vee\left(x_{1} * x_{2}\right) \vee \cdots \vee\left(x_{n-1} * x_{n}\right)=1
$$

Throughout this work $n$ will be fixed with $2 \leqslant n<\omega$, unless otherwise stated. The following result, which is also valid for Heyting algebras, is crucial. 
Proposition 1.1. Let $A$ be a Brouwerian algebra.

(i) $A \in \mathrm{S}_{\omega}$ if and only if for each prime filter $F$ of $A$ the set of prime filter containing $F$ forms a chain.

(ii) $A \in \mathrm{S}_{n}$ if and only if for each prime filter $F$ of $A$ the set of prime filters containing $F$ forms a chain with at most $n-1$ elements.

(iii) Let $g: A \rightarrow C_{n}^{1}$ be an onto map, and for $1 \leqslant i<n$ let $F_{i}=\left[c_{i}\right) g^{-1}$. Then $g$ is a homomorphism if and only if $F_{i}$ is a prime filter for $1 \leqslant i<n$ and the chain $F_{n-1} \subset F_{n-2} \subset \cdots \subset F_{1}$ is the set of all prime filters containing $F_{n-1}=1 g^{-1}$.

Proof. (i) See [7], [8], [22], [38] or [48].

(ii) See [24].

(iii) Since the map $g$ is onto, it is a homomorphism if and only if the unique Brouwerian-algebra congruence determined by the filter $F_{n-1}=1 g^{-1}$ has $\left\{A-F_{1}, F_{1}-F_{2}, F_{2}-F_{3}, \ldots, F_{n-2}-F_{n-1}, F_{n-1}\right\}$ as its set of congruence classes. If $F$ is a filter in a distributive lattice $D$, then the smallest congruence $\Psi^{F}$ on $D$ with $F$ as a congruence class is described as follows (see [6] or [44]):

$$
\langle a, b\rangle \in \Psi^{F} \Longleftrightarrow(a \wedge f=b \wedge f \text { for some } f \in F) \text {. }
$$

Let $P_{F}$ be the set of prime filters of $D$ containing $F$. It is well known and easily verified that

$$
\langle a, b\rangle \in \Psi^{F} \Longleftrightarrow\left(a \in P \Longleftrightarrow b \in P \text { for all } P \in P_{F}\right) \text {. }
$$

Thus it is sufficient to show that the unique Brouwerian-algebra congruence determined by a filter $F$ of $A$ coincides with the lattice congruence $\Psi^{F}$; but this is proved in W. C. Nemitz [39].

This proposition allows us to describe the Hom-sets of the form $\mathrm{S}_{n}\left(A, C_{n}^{1}\right)$ and $\mathrm{L}_{n}\left(A, C_{n}\right)$; in particular, we can describe the endomorphism monoids $\operatorname{End}\left(C_{n}^{1}\right)$ and $\operatorname{End}\left(C_{n}\right)$.

Let $e \in \operatorname{End}\left(C_{n}^{1}\right)$. Then $e$ is order preserving and there is a filter $\left[c_{k}\right)$ of $C_{n}^{1}$ such that $\left[c_{k}\right) e=\{1\}$ and, for all $i, j<k, c_{i} e=c_{j} e$ implies $i=j$.

Conversely, any map $e: C_{n}^{1} \rightarrow C_{n}^{1}$ with these properties is an endomorphism of $C_{n}^{1}$. It follows that

$$
\left|\operatorname{End}\left(C_{n}^{1}\right)\right|=\left(\begin{array}{c}
n-1 \\
0
\end{array}\right)+\left(\begin{array}{c}
n-1 \\
1
\end{array}\right)+\cdots+\left(\begin{array}{l}
n-1 \\
n-1
\end{array}\right)=2^{n-1}
$$

The endomorphisms of $C_{n}$ are determined similarly; the only additional restriction being $0 e=0$. By identifying $C_{n}^{1}$ with the filter $\left[c_{1}\right)$ of $C_{n+1}$ we obtain a one-to-one correspondence, in fact, a monoid isomorphism, between $\operatorname{End}\left(C_{n}^{1}\right)$ and 
$\operatorname{End}\left(C_{n+1}\right)$. Hence $\left|\operatorname{End}\left(C_{n}\right)\right|=2^{n-2}$. The identity map in both $\operatorname{End}\left(C_{n}^{1}\right)$ and $\operatorname{End}\left(C_{n}\right)$ is denoted by $\iota$; the zero of the monoid $\operatorname{End}\left(C_{n}^{1}\right)$, namely the retraction onto $\{1\}$, is denoted by $\theta$.

Definition 1.2. Let $A \in \mathrm{S}_{n}$ and let $F=F_{k} \subset F_{k-1} \subset \cdots \subset F_{1}$ be the chain of all prime filters containing the prime filter $F$. The homomorphism $g_{F} \in$ $\mathrm{S}_{n}\left(A, C_{n}^{1}\right)$ determined by $F$ is defined by

$$
a g_{F}= \begin{cases}1 & \text { if } a \in F=F_{k} \\ c_{i} & \text { if } a \in F_{i}-F_{i+1} \quad(1 \leqslant i<k) \\ 0 & \text { if } a \in A-F_{1}\end{cases}
$$

If $F$ is a filter of an algebra $A \in \mathrm{L}_{n}$, then $g_{F}$ is defined in exactly the same way. Proposition 1.1 guarantees that $g_{F}$ is well defined. In essence, $g_{F}$ maps all the elements of $F$ to 1 and all the other elements of $A$ as low as possible in the chain. The following factorization lemma will prove to be particularly useful.

LEMmA 1.3. (i) Let $g \in \mathrm{S}_{n}\left(A, C_{n}^{1}\right)$ and let $g_{\downarrow}$ be the homomorphism determined by $F=1 g^{-1}$. Then there exists $e \in \operatorname{End}\left(C_{n}^{1}\right)$ with $g=g_{\downarrow} e$.

(ii) Let $g \in \mathrm{L}_{n}\left(A, C_{n}\right)$ and let $g_{\downarrow}$ be the homomorphism determined by $F=1 g^{-1}$. Then there exists $e \in \operatorname{End}\left(C_{n}\right)$ with $g=g_{\downarrow} e$.

Proof. We only prove (i) since the proof of (ii) is almost identical. Let $F=F_{k} \subset F_{k-1} \subset \cdots \subset F_{1}$ be the chain of all prime filters containing $F$. Let $a_{0} \in A-F_{1}$, let $a_{i} \in F_{i}-F_{i+1}$, and define $e: C_{n}^{1} \rightarrow C_{n}^{1}$ by $c_{i} e=1$ for $k \leqslant i$ $<n$, and $c_{i} e=a_{i} g$ for $0 \leqslant i<k$. By Proposition 1.1 (iii), $g$ is constant on $A-$ $F_{1}, F_{i}-F_{i+1}(1 \leqslant i<k)$, and $F_{k}$. Thus $g=g_{\downarrow} e$; and $e$ is an endomorphism of $C_{n}^{1}$ since it is order preserving and, for all $i, j<k, c_{i} e=c_{j} e$ implies $i=j$.

A Boolean space is a zero-dimensional compact space, or equivalently, a compact space with a basis of clopen sets. The category of Boolean spaces and continuous maps is denoted by ZComp, and for $X, Y \in Z$ Comp, $C(X, Y)$ denotes the set of continuous maps from $X$ to $Y$. Recall that any closed subspace of a product of finite discrete spaces is a Boolean space, and hence $\mathrm{S}_{n}\left(A, C_{n}^{1}\right)$ and $\mathrm{L}_{n}\left(A, C_{n}\right)$ are Boolean spaces (regarded as subspaces of $\left(C_{n}^{1}\right)^{A}$ and $\left(C_{n}\right)^{A}$ respectively).

Let $X$ be a pointed Boolean space. Then the set $E^{1}(X)$ of point-preserving continuous maps $\varphi: X \rightarrow X$ is a monoid with id $_{X}$ as identity and the retraction onto the distinguished point as a zero. Let $\mathbf{X}_{n}$ be the category of pointed Boolean spaces which have a continuous action of the monoid End $\left(C_{n}^{1}\right)$ (that is, a semigroup homomorphism, $e \rightarrow \tilde{e}$, from $\operatorname{End}\left(C_{n}^{1}\right)$ into $E^{1}(X)$ such that $\tilde{\imath}=$ $\operatorname{id}_{X}$ ) for which $\tilde{\theta}$ is the retraction onto the distinguished point. A map $\psi \in$ $C(X, Y)$ is a morphism of $\mathrm{X}_{n}$ if it is a point preserving and preserves the action of $\operatorname{End}\left(C_{n}^{1}\right)$, that is, $x \tilde{e} \psi=x \psi \tilde{e}$ for all $x \in X$ and all $e \in \operatorname{End}\left(C_{n}^{1}\right)$. Observe 
that $C_{n}^{1} \in \mathrm{X}_{n}: 1$ is the distinguished point and for all $e \in \operatorname{End}\left(C_{n}^{1}\right), \tilde{e}=e$.

For all $A \in \mathrm{S}_{n}$ the Boolean space $\mathrm{S}_{n}\left(A, C_{n}^{1}\right)$ may be lifted to an object of $\mathrm{X}_{n}$ : the constant map $\hat{1}: A \rightarrow C_{n}^{1}$ onto $\{1\}$ is the distinguished point and for all $e \in \operatorname{End}\left(C_{n}\right), \tilde{e} \in E^{1}\left(\mathrm{~S}_{n}\left(A, C_{n}^{1}\right)\right)$ is defined by $g \tilde{e}=g e$. If $h \in \mathrm{S}_{n}(A, B)$, then it is clear that

$$
\mathrm{S}_{n}\left(h, C_{n}^{1}\right): \mathrm{S}_{n}\left(B, C_{n}^{1}\right) \rightarrow \mathrm{S}_{n}\left(A, C_{n}^{1}\right),
$$

defined by. $g \mathrm{~S}_{n}\left(h, C_{n}^{1}\right)=h g$, is a morphism of $\mathrm{X}_{n}$; whence $\mathrm{S}_{n}\left(-, C_{n}^{1}\right): \mathrm{S}_{n} \rightarrow$ $\mathbf{X}_{n}^{\text {op }}$ is a well-defined functor. It is also easy to verify that for all $X \in \mathbf{X}_{n}$, $\mathrm{X}_{n}\left(X, C_{n}^{1}\right)$ is a subalgebra of $\left(C_{n}^{1}\right)^{X}$, and that for all $\psi \in \mathrm{X}_{n}(X, Y)$,

$$
\mathrm{X}_{n}\left(\psi, C_{n}^{1}\right): \mathrm{X}_{n}\left(Y, C_{n}^{1}\right) \rightarrow \mathrm{X}_{n}\left(X, C_{n}^{1}\right),
$$

defined by $\varphi \mathrm{X}_{n}\left(\psi, C_{n}^{1}\right)=\psi \varphi$, is a homomorphism; whence $\mathrm{X}_{n}\left(-, C_{n}^{1}\right): \mathbf{X}_{n}^{\text {op }} \rightarrow$ $S_{n}$ is a well-defined functor.

In the next section we shall show that $S_{n}$ and $X_{n}$ are dual categories; the next result paves the way. For each $A \in \mathrm{S}_{n}$ define $\eta_{A}: A \rightarrow \mathrm{X}_{n}\left(\mathrm{~S}_{n}\left(A, C_{n}^{1}\right), C_{n}^{1}\right)$ by $a \eta_{A}=\Gamma_{a}$, where $g \Gamma_{a}=a g$ for all $g \in \mathrm{S}_{n}\left(A, C_{n}^{1}\right)$; for each $X \in \mathrm{X}_{n}$ define $\epsilon_{X}: X \rightarrow S_{n}\left(X_{n}\left(X, C_{n}^{1}\right), C_{n}^{1}\right)$ by $x \epsilon_{X}=\Gamma_{x}$, where $\varphi \Gamma_{x}=x \varphi$ for all $\varphi \in$ $\mathbf{X}_{n}\left(X, C_{n}^{1}\right)$.

Proposition 1.4. $\left\langle\mathrm{S}_{n}\left(-, C_{n}^{1}\right), \mathrm{X}_{n}\left(-, C_{n}^{1}\right) ; \eta, \epsilon\right\rangle$ is an adjunction from $\mathrm{S}_{n}$ to $X_{n}^{o p}$.

Proof. By [37, Theorem 2, p. 81] it is sufficient to prove that $\eta$ is a natural transformation and that each $\eta_{A}$ is universal to $\mathrm{X}_{n}\left(-, C_{n}^{1}\right)$ from $A$. We will only establish the universal mapping property since a simple calculation shows that $\eta$ is a well-defined natural transformation. Let $A \in \mathrm{S}_{n}, X \in \mathrm{X}_{n}$, and let $h: A \rightarrow \mathrm{X}_{n}\left(X, C_{n}^{1}\right)$ be a homomorphism. If $\psi: X \rightarrow \mathrm{S}_{n}\left(A, C_{n}^{1}\right)$ satisfies $\eta_{A} \mathrm{X}_{n}\left(\psi, C_{n}^{1}\right)=h$, then $\psi$ must be given by $a(x \psi)=x(a h)$, and hence we must prove that this defines a morphism of $X_{n}$. But, since each of the maps ah ( $a \in$ $A)$ is continuous, point preserving, and preserves the action of $\operatorname{End}\left(C_{n}^{1}\right)$, it follows immediately that $\psi$ is continuous and point preserving, and that for each $e \in$ $\operatorname{End}\left(C_{n}^{1}\right), a(x \tilde{e} \psi)=x \tilde{e}(a h)=(x(a h)) \tilde{e}=(a(x \psi)) \tilde{e}=a(x \psi \tilde{e})$; whence $\psi$ preserves the action of $\operatorname{End}\left(C_{n}^{1}\right)$.

Let $X$ be a Boolean space. Then the set $E(X)=C(X, X)$ is a monoid with id ${ }_{X}$ as identity. Let $Y_{n}$ be the category of Boolean spaces which have a continuous action, $e \rightarrow \tilde{e}$, of the monoid $\operatorname{End}\left(C_{n}\right)$; the morphisms of $Y_{n}$ being the continuous maps which preserve the action of $\operatorname{End}\left(C_{n}\right)$. Observe that $C_{n} \in$ $Y_{n}:$ for all $e \in \operatorname{End}\left(C_{n}\right), \tilde{e}=e$. The Hom-functors $\mathrm{L}_{n}\left(-, C_{n}\right): \mathrm{L}_{n} \rightarrow \mathrm{Y}_{n}^{\text {op }}$ and $Y_{n}\left(-, C_{n}\right): Y_{n}^{o p} \rightarrow L_{n}$ are defined exactly as they were for $S_{n}$ and $X_{n}$. 
Clearly the analogue of Proposition 1.4 holds for $L_{n}$ and $Y_{n}$. As before, for each $A \in \mathrm{L}_{n}$ define $\eta_{A}: A \rightarrow \mathrm{Y}_{n}\left(\mathrm{~L}_{n}\left(A, C_{n}\right), C_{n}\right)$ by $a \eta_{A}=\Gamma_{a}$, where $g \Gamma_{a}$ $=a g$ for all $g \in \mathrm{L}_{n}\left(A, C_{n}\right)$, and for each $X \in \mathrm{Y}_{n}$ define

$$
\epsilon_{X}: X \rightarrow \mathrm{L}_{n}\left(\mathrm{Y}_{n}\left(X, C_{n}\right), C_{n}\right)
$$

by $x \epsilon_{X}=\Gamma_{x}$, where $\varphi \Gamma_{x}=x \varphi$ for all $\varphi \in Y_{n}\left(X, C_{n}\right)$.

Proposition 1.5. $\left\langle\mathrm{L}_{n}\left(-, C_{n}\right), \mathrm{Y}_{n}\left(-, C_{n}\right) ; \eta, \epsilon\right\rangle$ is an adjunction from $\mathrm{L}_{n}$ to $Y_{n}^{O p}$.

The proofs of the duality theorems pivot around the following simple result.

LEMMA 1.6. (i) If $\varphi \in \mathrm{X}_{n}\left(\mathrm{~S}_{n}\left(A, C_{n}^{1}\right), C_{n}^{1}\right)$, then $g \varphi \in \operatorname{Im}(g)$ for all $g \in$ $\mathrm{S}_{n}\left(A, C_{n}^{1}\right)$.

(ii) If $\varphi \in \mathrm{Y}_{n}\left(\mathrm{~L}_{n}\left(A, C_{n}\right), C_{n}\right)$, then $g \varphi \in \operatorname{Im}(g)$ for all $g \in \mathrm{L}_{n}\left(A, C_{n}\right)$.

Proof. We only prove (i). Since $\varphi$ preserves the action of $\operatorname{End}\left(C_{n}^{1}\right)$, by Lemma 1.3 it is sufficient to show that $g_{\downarrow} \varphi \in \operatorname{Im}\left(g_{\downarrow}\right)$ for all $g \in \mathrm{S}_{n}\left(A, C_{n}^{1}\right)$. Without loss of generality, assume that $g$ is not the constant homomorphism $\hat{1}$. Let $\operatorname{Im}\left(g_{\downarrow}\right)=\left(c_{k-1}\right] \cup\{1\}, 1 \leqslant k<n$, and let $e_{k}$ be the endomorphism of $C_{n}^{1}$ determined by the prime filter $\left[c_{k}\right)$. Clearly $g_{\downarrow}=g_{\downarrow} e_{k}=g_{\downarrow} \tilde{e}_{k}$ and hence $g_{\downarrow} \varphi=g_{\downarrow} \tilde{e}_{k} \varphi=g_{\downarrow} \varphi \widetilde{e}_{k}=g_{\downarrow} \varphi e_{k} ;$ whence $g_{\downarrow} \varphi \in \operatorname{Im}\left(e_{k}\right)=\operatorname{Im}\left(g_{\downarrow}\right)$.

2. The dualities. Since we are primarily interested in representing algebras as algebras of continuous functions, our emphasis is on dualities rather than full dualities, in the following sense.

Definition 2.1. Let $\mathbf{A}$ and $\mathbf{X}$ be categories and assume that $D: A \rightarrow X^{\text {op }}$ is left adjoint to $E: \mathbf{X}^{\mathbf{o p}} \rightarrow \mathbf{A}$. Then $\langle D, E\rangle$ is a duality (between $\mathbf{A}$ and $\mathbf{X}$ ) if the unit $\eta: \mathrm{id}_{\mathrm{A}} \rightarrow E D$ of the adjunction is a natural isomorphism, and is a full duality if the counit $\epsilon: \mathrm{id}_{\mathbf{X}} \rightarrow D E$ is also a natural isomorphism.

Firstly, we will establish the duality between $L_{n}$ and $Y_{n}$; for the duality between $S_{n-1}$ and $X_{n-1}$ will then follow. In order to do so we require H. A. Priestley's duality for bounded distributive lattices.

A subset $U$ of a poset $X$ is increasing if $x \in U$ and $y \geqslant x$ imply that $y \in U$. A partially ordered, topological space $X$ is totally order disconnected if for all $x, y \in X$ with $x \notin y$ there exists a clopen increasing subset $U$ of $X$ such that $x \in U$ and $y \notin U$. The category of compact totally order-disconnected spaces and continuous order-preserving maps is denoted by $\mathbf{P}$. (Note that the underlying space of an object in $\mathbf{P}$ is a Boolean space.) The category of distributive lattices with zero and unit is denoted by $\mathrm{D}$. For each $A \in \mathrm{D}$ let $\chi(A)$ be the set of all prime filters of $A$, and for each $a \in A$ let $\mathfrak{X}_{a}=\{x \in X(A) \mid a \in x\}$. Order $\mathfrak{X}(A)$ by inclusion and let $\left\{X_{a} \mid a \in A\right\} \cup\left\{X(A)-X_{a} \mid a \in A\right\}$ be a basis for a topology on $\mathfrak{X}(A)$. Then $\mathfrak{X}(A) \in \mathrm{P}$. If $h \in \mathrm{D}(A, B)$, then $\mathfrak{X}(h): \mathfrak{X}(B) \rightarrow \mathfrak{X}(A)$ is defined by $x \mathcal{X}(h)=x h^{-1}$. For each $X \in \mathrm{P}$ let $\mathscr{Q}(X)$ be the lattice of clopen increasing 
subsets of $X$ with set union and intersection as operations. If $\psi \in P(X, Y)$, then $\mathscr{U}(\psi): \mathfrak{U}(Y) \rightarrow \mathscr{U}(X)$ is defined by $U \mathscr{U}(\psi)=U \psi^{-1}$. Both $X: \mathrm{D} \rightarrow \mathrm{p}^{\text {op }}$ and $\mathcal{U}: \mathrm{Pop} \rightarrow \mathrm{D}$ are well-defined functors. For each $A \in \mathrm{D}$ define $\eta_{A}: A \rightarrow$ QX $X(A)$ by $a \eta_{A}=X_{a}$, and for each $X \in P$ define $\epsilon_{X}: X \rightarrow X \mathscr{U}(X)$ by $x \epsilon_{X}=$ $\{U \in \mathscr{U}(X) \mid x \in U\}$; then $\langle X, \mathscr{Q} ; \eta, \epsilon\rangle$ is an adjunction from $D$ to $P$ op.

Theorem 2.2 (H. A. Priestley [40], [41]). $\langle\mathfrak{X}, \mathfrak{U}\rangle$ is a full duality between $\mathrm{D}$ and $\mathrm{P}$.

REMARK 2.3. If $A$ is a finite distributive lattice, then $X(A)$ is discretely topologized and hence $A$ is isomorphic to the lattice of increasing subsets of the poset $\mathfrak{X}(A)$ of its prime filters.

With this tool we may now establish the duality between $L_{n}$ and $Y_{n}$.

THEOREM 2.4. $\left\langle\mathrm{L}_{n}\left(-, C_{n}\right), \mathrm{Y}_{n}\left(-, C_{n}\right)\right\rangle$ is a duality between $\mathrm{L}_{n}$ and $\mathrm{Y}_{n}$.

Proof. Let $A \in \mathbf{L}_{n}$. Since each pair of distinct elements of a distributive lattice can be separated by a prime filter, it follows, by Proposition 1.1, that $\mathrm{L}\left(A, C_{n}\right)$ separates the points of $A$ and hence $\eta_{A}$ is an embedding. We now show that $\eta_{A}$ is also a surjection and hence is an isomorphism.

Define an equivalence relation $R$ on $\mathrm{L}_{n}\left(A, C_{n}\right)$ by $\langle g, h\rangle \in R \Longleftrightarrow 1 g^{-1}=$ $1 h^{-1}$ and note that $[g] R=\left[g_{\downarrow}\right] R$, where $[g] R$ denotes the equivalence class of $g$ in $\mathbf{L}_{n}\left(A, C_{n}\right) / R$. Define a partial order $\leqslant$ on the quotient space by $[g] R \leqslant$ $[h] R \Longleftrightarrow 1 g^{-1} \subseteq 1 h^{-1}$. Observe that

$[g] R \leqslant[h] R \Longleftrightarrow g_{\downarrow} \leqslant h_{\downarrow}$ (pointwise) $\Longleftrightarrow g_{\downarrow}=h_{\downarrow} e$ for some $e \in \operatorname{End}\left(C_{n}\right)$.

We claim that $\mathrm{L}_{n}\left(A, C_{n}\right) / R$ is homeomorphic and order isomorphic to $X(A)$. Define $G: \mathrm{L}_{n}\left(A, C_{n}\right) \rightarrow \mathfrak{X}(A)$ by $g G=1 g^{-1}$. The map $G$ is continuous since it is clear that the preimages under $G$ of the basic open sets $X_{a}$ and $X(A)-\mathfrak{X}_{a}$ are open in $\mathrm{L}_{n}\left(A, C_{n}\right)$. Since $G$ is constant on the equivalence classes of $R$ it induces a homeomorphism $\bar{G}$ between $\mathrm{L}_{n}\left(A, C_{n}\right) / R$ and $X(A)$ (see [13, Corollary 2.2, p. 227]). Furthermore,

$$
[g] R \leqslant[h] R \Longleftrightarrow 1 g^{-1} \subseteq 1 h^{-1} \Longleftrightarrow g G \leqslant h G \Longleftrightarrow([g] R) \bar{G} \leqslant([h] R) \bar{G} .
$$

and hence $\bar{G}$ is an order-isomorphism.

If $\varphi \in \mathrm{Y}_{n}\left(\mathrm{~L}_{n}\left(A, C_{n}\right), C_{n}\right)$, then $U=1 \varphi^{-1}$ is a clopen subset of $\mathrm{L}_{n}\left(A, C_{n}\right)$. If $g \in U$ and $\langle g, h\rangle \in R$, then $h \in U$. Indeed, let $g=g_{\downarrow} e$ and $h=h_{\downarrow} f$ be factorizations of $g$ and $h$ via Lemma 1.3; then $g_{\downarrow}=h_{\downarrow}$. Since $g \varphi=1$ we have $g_{\downarrow} \varphi e=g_{\downarrow} e \varphi=g \varphi=1$; but $g_{\downarrow} \varphi \in \operatorname{Im}\left(g_{\downarrow}\right)$ (Lemma 1.6) and therefore $g_{\downarrow} \varphi=1$. Hence $h \varphi=h_{\downarrow} f \varphi=h_{\downarrow} \varphi f=g_{\downarrow} \varphi f=1 f=1$, and so $h \in U$. Thus the clopen set $U$ is a union of $R$-equivalence classes and consequently $U / R=\{[g] R \mid g \in U\}$ is clopen in $\mathrm{L}_{n}\left(A, C_{n}\right) / R$. Assume that $[g] R \in U / R$. If $[g] R \leqslant[h] R$, then there exists $e \in \operatorname{End}\left(C_{n}\right)$ with $g_{\downarrow} e=h_{\downarrow}$, and thus $h_{\downarrow} \varphi=g_{\downarrow} e \varphi=g_{\downarrow} \varphi e=1 e=1$. Hence 
$h_{\downarrow} \in U$ and so $[h] R=\left[h_{\downarrow}\right] R \in U / R$. We have shown that $U / R$ is a clopen increasing subset of $\mathrm{L}_{n}\left(A, C_{n}\right) / R$.

Since $\bar{G}: \mathbf{L}_{n}\left(A, C_{n}\right) / R \rightarrow \mathfrak{X}(A)$ is an isomorphism in $\mathbf{P}$, by Theorem 2.2 there exists $a \in A$ such that $(U / R) \bar{G}=\mathfrak{X}_{a}$, that is,

$$
\text { for all } g \in \mathrm{L}_{n}\left(A, C_{n}\right), g \varphi=1 \text { if and only if } a g=1 \text {, }
$$

We claim that $a \eta_{A}=\varphi$, that is, for all $g \in \mathrm{L}_{n}\left(A, C_{n}\right), a g=g \varphi$.

By Lemma 1.3 it is sufficient to prove that for all $g \in \mathrm{L}_{n}\left(A, C_{n}\right), a g_{\downarrow}=$ $g_{\downarrow} \varphi$. Let $1 g^{-1}=F_{k} \subset F_{k-1} \subset \cdots \subset F_{1}$ be the chain of all prime filters containing $1 g^{-1}$. For $1 \leqslant i \leqslant k$, let $g_{i}: A \rightarrow C_{n}$ be the homomorphism determined by the prime filter $F_{i}$, let $e_{i} \in \operatorname{End}\left(C_{n}\right)$ be the endomorphism determined by the prime filter $\left[c_{i}\right)$, and observe that $g_{i}=g_{\downarrow} e_{i}$.

If $a \in F_{k}=1 g^{-1}$, then $a g_{\downarrow}=1=g_{\downarrow} \varphi$ by (*). If $a \in A-F_{1}$, then $a g_{1} \neq$ 1 and hence $g_{1} \varphi \neq 1$ by (*). But $g_{1} \varphi \in \operatorname{Im}\left(g_{1}\right)$ by Lemma 1.6, and hence $g_{1} \varphi$ $=0$. Consequently $g_{\downarrow} \varphi e_{1}=g_{\downarrow} e_{1} \varphi=g_{1} \varphi=0$, and so $g_{\downarrow} \varphi=0$. But, since $a \in$ $A-F_{1}$, we also have $a g_{\downarrow}=0$; whence $a g_{\downarrow}=0=g_{\downarrow} \varphi$. Finally, assume that $a \in$ $F_{l}-F_{l+1}, 1 \leqslant l<k$. Cearly $a g_{l}=1$ and $a g_{l+1} \neq 1$. Thus by $(*), g_{l} \varphi=1$ and $g_{l+1} \varphi \neq 1$. Hence $g_{\downarrow} \varphi e_{l}=g_{\downarrow} e_{l} \varphi=g_{l} \varphi=1$ and $g_{\downarrow} \varphi e_{l+1}=g_{\downarrow} e_{l+1} \varphi=g_{l+1} \varphi \neq 1$, that is, $g_{\downarrow} \varphi \in\left[c_{l}\right)-\left[c_{l+1}\right)=\left\{c_{l}\right\}$. Hence $g_{\downarrow} \varphi=c_{l}$. But, since $a \in F_{l}-F_{l+1}$, we also have $a g_{\downarrow}=c_{l}$; whence $a g_{\downarrow}=c_{l}=g_{\downarrow} \varphi$.

For $n=2$ the duality reduces to M. H. Stone's duality for Boolean algebras ([45], see also [23]) and hence is full.

For $n=3$ the duality is also full; we sketch a proof. By Proposition 1.1, for all $A \in \mathrm{L}_{3}$, every prime filter $x \in \mathfrak{X}(A)$ induces a homomorphism $g_{x} \in$ $\mathrm{L}_{3}\left(A, C_{3}\right)$, namely the homomorphism determined by $x$, and conversely, every homomorphism $g \in \mathrm{L}_{3}\left(A, C_{3}\right)$ is uniquely determined by the filter $x=1 g^{-1}$. For all $x \in \mathfrak{X}(A)$ let $x \tilde{e}_{1}$ be the unique maximal filter containing $x$. Then $\tilde{e}_{1}$ : $X(A) \rightarrow X(A)$ is continuous, $X(A) \in Y_{3}$, and the one-to-one correspondence described above is a $Y_{3}$-isomorphism between $X(A)$ and $\mathbf{L}_{3}\left(A, C_{3}\right)$. Define a partial order $\leqslant$ on each $X \in Y_{3}$ by $x \leqslant y \Longleftrightarrow\left(x=y\right.$ or $\left.x \tilde{e}_{1}=y\right)$. Under this partial order $X$ is totally order disconnected, that is, $X \in P$. Every clopen increasing subset $U$ of $X$ determines a map $\varphi_{U} \in \mathrm{Y}_{3}\left(X, C_{3}\right)$ :

$$
x \varphi_{U}= \begin{cases}1 & \text { if } x \in U, \\ c_{1} & \text { if } x \in\left(U \tilde{e}_{1}^{-1}\right)-U, \\ 0 & \text { if } x \notin U \tilde{e}_{1}^{-1},\end{cases}
$$

and conversely, every map $\varphi \in Y_{3}\left(X, C_{3}\right)$ is uniquely determined by the clopen increasing subset $U=1 \varphi^{-1}$. This one-to-one correspondence is an $\mathbf{L}_{3}$-isomorphism 


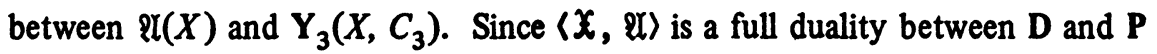
it follows that $\left\langle\mathrm{L}_{3}\left(-, C_{3}\right), \mathrm{Y}_{3}\left(-, C_{3}\right)\right\rangle$ is a full duality between $\mathrm{L}_{3}$ and $\mathrm{Y}_{3}$. (An alternative proof may be obtained by applying the duality for Stone algebras developed in [9] and [42].)

For $n \geqslant 4$ the duality is not full. Let $X=\{0,1\}$, let $\tilde{\imath}=\mathrm{id}_{X}$ and for all $e \neq \iota$ let $\tilde{e}$ be the retraction onto the point 1 . It is easily checked that the action of $\operatorname{End}\left(C_{n}\right)$ is well defined and that $Y_{n}\left(X, C_{n}\right)=\left\{\varphi_{0}, \varphi_{1}, \varphi_{2}\right\}$, where $0 \varphi_{0}=$ $1 \varphi_{0}=0,0 \varphi_{1}=0$ and $1 \varphi_{1}=c_{n-2}$, and $0 \varphi_{2}=1 \varphi_{2}=1$. Hence $Y_{n}\left(X, C_{n}\right) \cong$ $C_{3}$, which gives

$$
\left|\mathrm{L}_{n}\left(\mathrm{Y}_{n}\left(X, C_{n}\right), C_{n}\right)\right|=\left|\mathrm{L}_{n}\left(C_{3}, C_{n}\right)\right|=n-1 \neq 2
$$

whence $\epsilon_{X}$ is not a surjection.

We turn now to $S_{n}$ and $X_{n}$.

THEOREM 2.5. $\left\langle\mathrm{S}_{n}\left(-, C_{n}^{1}\right), \mathrm{X}_{n}\left(-, C_{n}^{1}\right)\right\rangle$ is a duality between $\mathrm{S}_{n}$ and $\mathrm{X}_{n}$.

Proof. If $A \in \mathrm{S}_{n}$, then ${ }_{0} A$, the Heyting algebra obtained by adjoining a new zero to $A$, is an object of $\mathrm{L}_{n+1}$ by Proposition 1.1. If $g \in \mathrm{S}_{n}\left(A, C_{n}^{1}\right)$, then identifying $C_{n}^{1}$ with the filter $\left[c_{1}\right)$ of $C_{n+1}$, we obtain ${ }_{0} g \in \mathrm{L}_{n+1}\left({ }_{0} A, C_{n+1}\right)$ by extending $g$ in the obvious manner. Since $\operatorname{End}\left(C_{n}^{1}\right) \cong \operatorname{End}\left(C_{n+1}\right)$ it follows that $\mathrm{S}_{n}\left(A, C_{n}^{1}\right) \cong \mathrm{L}_{n+1}\left({ }_{0} A, C_{n+1}\right)$, where the distinguished point of $\mathrm{L}_{n+1}\left({ }_{0} A, C_{n+1}\right)$ is the homomorphism $h_{1}:{ }_{0} A \rightarrow C_{n+1}$ determined by the prime filter $A$. Note that for all $g \in \mathrm{L}_{n+1}\left({ }_{0} A, C_{n+1}\right), g e_{1}=h_{1}$. If

$$
\varphi \in \mathrm{Y}_{n+1}\left(\mathrm{~L}_{n+1}\left({ }_{0} A, C_{n+1}\right), C_{n+1}\right),
$$

then $h_{1} \varphi \in \operatorname{Im}\left(h_{1}\right)=\{0,1\}$ by Lemma 1.6. If $h_{1} \varphi=0$, then for all $g \in$ $\mathbf{L}_{n+1}\left({ }_{0} A, C_{n+1}\right)$ we have $g \varphi e_{1}=g e_{1} \varphi=h_{1} \varphi=0$ and so $g \varphi=0$, that is, $\varphi=$ 0 , the identically zero map. Similarly, if $h_{1} \varphi=1$, then for all $g \in \mathrm{L}_{n+1}\left({ }_{0} A, C_{n+1}\right)$, $g \varphi \in\left[c_{1}\right)$. It follows readily that

$$
\mathrm{Y}_{n+1}\left(\mathrm{~L}_{n+1}\left({ }_{0} A, C_{n+1}\right), C_{n+1}\right) \cong \mathbf{X}_{n}\left(\mathrm{~L}_{n+1}\left({ }_{0} A, C_{n+1}\right), C_{n}^{1}\right) \cup\{\hat{0}\}
$$

Thus

$$
\begin{aligned}
\mathrm{X}_{n}\left(\mathrm{~S}_{n}\left(A, C_{n}^{1}\right), C_{n}^{1}\right) \cong \mathrm{X}_{n}\left(\mathrm{~L}_{n+1}\left({ }_{0} A, C_{n+1}\right), C_{n}^{1}\right) \\
\cong \mathrm{Y}_{n}\left(\mathrm{~L}_{n+1}\left({ }_{0} A, C_{n+1}\right), C_{n+1}\right)-\{\hat{0}\} \cong{ }_{0} A-\{0\}=A .
\end{aligned}
$$

For $n=2$ the duality is full. A dual generalized Boolean algebra (DGBA) is a distributive lattice with unit in which each principal filter is a Boolean algebra. It is well known that a DGBA is a Brouwerian algebra and that $S_{2}$ is the 
class of all DGBA's (see [29]). Since $\operatorname{End}\left(C_{2}^{1}\right)=\{\imath, \theta\}$, the action of $\operatorname{End}\left(C_{2}^{1}\right)$ is trivial and hence $X_{2}$ is isomorphic to the category of pointed Boolean spaces. For each $X \in \mathrm{X}_{2}$ define the action of $\operatorname{End}\left(C_{3}\right)$ on $X$ by declaring that $\tilde{e}_{1}=\tilde{\theta}$, the retraction onto the distinguished point. Then $X \in Y_{3}$ and it is easily seen that $Y_{3}\left(X, C_{3}\right) \cong{ }_{0}\left[X_{2}\left(X, C_{2}^{1}\right)\right]$. Hence, since the duality between $L_{3}$ and $Y_{3}$ is full, we have

$$
\begin{aligned}
\mathrm{S}_{2}\left(\mathrm{X}_{2}\left(X, C_{2}^{1}\right), C_{2}^{1}\right) & \cong \mathrm{L}_{3}\left({ }_{0}\left[\mathrm{X}_{2}\left(X, C_{2}^{1}\right)\right], C_{3}\right) \\
& \cong \mathrm{L}_{3}\left(\mathrm{Y}_{3}\left(X, C_{3}\right), C_{3}\right) \cong X,
\end{aligned}
$$

and thus the duality between $\mathbf{S}_{\mathbf{2}}$ and $\mathbf{X}_{\mathbf{2}}$ is full.

For $n \geqslant 3$ the duality is not full. Again let $X=\{0,1\}$, let $\tilde{\imath}=\mathrm{id}_{X}$ and for $e \neq \imath$ let $\tilde{e}$ be the retraction onto the distinguished point 1 . The action of $\operatorname{End}\left(C_{n}^{1}\right)$ is well defined and $X_{n}\left(X, C_{n}^{1}\right)=\left\{\varphi_{0}, \varphi_{1}\right\}$, where $0 \varphi_{0}=c_{n-2}$ and $1 \varphi_{0}=1$, and $0 \varphi_{1}=1 \varphi_{1}=1$. Hence

$$
\left|\mathrm{S}_{n}\left(\mathrm{X}_{n}\left(X, C_{n}^{1}\right), C_{n}^{1}\right)\right|=\left|\mathrm{S}_{n}\left(C_{2}^{1}, C_{n}^{1}\right)\right|=n \neq 2,
$$

and thus $\epsilon_{X}$ is not a surjection.

3. ZComp-free functors and sur-projectives in $X_{n}$ and $Y_{n}$. If there is a faithful functor $|-|: X \rightarrow C$, then $\mathbf{X}$ is grounded in $\mathbf{C}$ and $|-|$ is called a grounding. A category $\mathbf{X}$ has a $\mathbf{C}$-free functor if it is grounded in $\mathbf{C}$ and the grounding has a left adjoint $\mathfrak{F}: \mathbf{C} \rightarrow \mathbf{X}$. If $\mathbf{C}=$ Set, then $\mathfrak{F}$ is simply called a free functor. Forgetful functors are the most accessible examples of groundings and the formation of free algebras in an equation class is a typical example of a free functor.

If $|-|: X \rightarrow$ Set is a grounding, then $\varphi \in \mathbf{X}$ is a surjection if $|\varphi|$ is onto. An object $P \in \mathrm{X}$ is sur-projective in $\mathrm{X}$ if for every surjection $\varphi: X \rightarrow Y$ and every morphism $\psi: P \rightarrow Y$ there exists $\psi^{\prime}: P \rightarrow X$ with $\psi^{\prime} \varphi=\psi$. Recall that $X$ is a retract of $Y$ if there exist morphisms $\varphi: X \rightarrow Y$ and $\psi: Y \rightarrow X$ in $X$ with $\varphi \psi=\mathrm{id}_{X}$.

The following result, which is proved in [25], illustrates the importance of ZComp-free functors.

PROPOSITION 3.1. If $\mathbf{X}$ is a category grounded in ZComp and of: ZComp $\rightarrow \mathrm{X}$ is a ZComp-free functor, then the following are equivalent:

(i) $P$ is sur-projective in $\mathrm{X}$;

(ii) $P$ is a retract of $\mathcal{F}(\beta S)$ for some set $S$ where $\beta$ : Set $\rightarrow Z$ Comp is the Stone-Čech compactification functor;

(iii) $P$ is a retract of $\mathfrak{F}(X)$ for some compact extremally disconnected space $X$.

$X_{n}$ and $Y_{n}$ are grounded in ZComp by the forgetful functors and we now describe their ZComp-free functors. As a preliminary we prove a purely universalalgebraic result. 
As before, if $A$ is an algebra and $B$ is a subalgebra of $A^{X}$, then for each $x \in X$ the map $\Gamma_{x}: B \rightarrow A$ is defined by $\varphi \Gamma_{x}=x \varphi$ for all $\varphi \in B$. For all $a \in A$, $\hat{a}: X \rightarrow A$ denotes the constant map onto $\{a\}$. The monoid of endomorphisms of $A$ is denoted by $\operatorname{End}(A)$ and the constant endomorphism onto a one-element subalgebra $\{a\}$ of $A$ is denoted by $\bar{a}$.

Proposition 3.2. Let $A$ be a nontrivial finite algebra all of whose nontrivial subalgebras are subdirectly irreducible and assume that every algebra in the equational class $\mathrm{A}=\operatorname{HSP}(\{A\})$ generated by $A$ has a distributive congruence lattice. If $X$ is a Boolean space and $B$ is a subalgebra of $C(X, A)$ containing the constant maps, then every homomorphism $g \in \mathbf{A}(B, A)$ is of the form $\Gamma_{x}$ e for some $x \in X$ and some $e \in \operatorname{End}(A)$.

Proof. Let $g \in \mathbf{A}(B, A)$. If $\operatorname{Im}(g)=\{a\}$, then choose $x \in X$ arbitrarily; clearly $g=\Gamma_{x} \bar{a}$. If $\operatorname{Im}(g)$ is nontrivial then it is subdirectly irreducible, and by Jónsson's lemma [28, Lemma 3.1, p. 114] there is an maximal filter $F$ of the Boolean algebra of all subsets of $X$ with $\Theta_{F} \mid B \leqslant \operatorname{Ker}(g)$, where $\Theta_{F}$ is the congruence on $A^{X}$ given by

$$
\langle\varphi, \psi\rangle \in \Theta_{F} \Leftrightarrow(\mathrm{Eq}(\varphi, \psi)=\{x \in X \mid x \varphi=x \psi\} \in F) .
$$

Let $F^{\prime}=\left\{U \in F \mid U\right.$ is clopen in $X$. Then $F^{\prime}$ is a maximal filter of the Boolean algebra of clopen subsets of $X$, and hence, since $X$ is a Boolean space, there exists (a unique) $x \in X$ such that $F^{\prime}=\{U \mid U$ is clopen in $X$ and $x \in U\}$.

Now $\operatorname{Eq}(\varphi, \psi)=\bigcup\left(a \varphi^{-1} \cap a \psi^{-1} \mid a \in A\right)$, and thus if $\varphi, \psi \in \mathcal{C}(X, A)$, then $\mathrm{Eq}(\varphi, \psi)$ is clopen in $X$. Hence

$$
\langle\varphi, \psi\rangle \in \Theta_{F} \mid B \Leftrightarrow \mathrm{Eq}(\varphi, \psi) \in F^{\prime} \Longleftrightarrow x \varphi=x \psi .
$$

Define $e: A \rightarrow A$ by $a e=\hat{a} g$. Since $B$ contains the set $\{\hat{a} \mid a \in A\}$ of constant maps, $e$ is well defined, and since $A$ is isomorphic to $\{\hat{a} \mid a \in A\}, e$ is an endomorphism. We claim that $g=\Gamma_{x} e$. If $\varphi \in B$, then $\varphi\left(\Gamma_{x} e\right)=x \varphi e=(\widehat{x \varphi}) g$. $\operatorname{But}\langle\varphi,(\widehat{x \varphi})\rangle \in \Theta_{F} \mid B$ since $x \varphi=x(\widehat{x \varphi})$, and hence $\langle\varphi,(\widehat{x \varphi})\rangle \in \operatorname{Ker}(g)$ since $\Theta_{F} \mid B \leqslant \operatorname{Ker}(g)$. Thus $\varphi g=(\widehat{x \varphi}) g$, and consequently $\varphi\left(\Gamma_{x} e\right)=\varphi g$.

It is readily verified that for any algebra $B$ of the same type as $A, \mathrm{~A}(B, A)$ is a closed subspace of $A^{B}$, and hence $\mathrm{A}(B, A)$ is a Boolean space since $A$ is finite.

Corollary 3.3. Assume that the conditions of the proposition hold and that $\left\{a_{0}, \ldots, a_{n-1}\right\}$ is the set of painwise distinct elements which form one-element subalgebras of $A$. Let

$$
\mathfrak{F}(X)=\left(X \times\left(\operatorname{End}(A)-\left\{\bar{a}_{0}, \ldots, \bar{a}_{n-1}\right\}\right)\right) \dot{\cup}\left\{\bar{a}_{0}, \ldots, \bar{a}_{n-1}\right\}
$$

and define $\mu_{X}: \mathfrak{f}(X) \rightarrow \mathrm{A}(C(X, A), A)$ by $\langle x, e\rangle \mu_{X}=\Gamma_{x} e$ and $\bar{a}_{i} \mu_{X}=\hat{a}_{i}$ 
$(i<n)$. Then $\mu_{X}$ is a homeomorphism of $₹(X)$ onto $\mathrm{A}(\mathrm{C}(X, A), A)$.

Proof. The proposition guarantees that $\mu_{X}$ is onto; we now show that it is one-to-one. Let $\langle x, e\rangle,\langle y, f\rangle \in\{\}(X)$. If $e \neq f$, then there exists $a \in A$ with $a e \neq a f$, and consequently

$$
\hat{a}\left(\langle x, e\rangle \mu_{X}\right)=\hat{a} \Gamma_{x} e=a e \neq a f=\hat{a} \Gamma_{y} f=\hat{a}\left(\langle y, f\rangle \mu_{X}\right) .
$$

If $e=f$ and $x \neq y$, then let $U$ be a clopen subset of $X$ with $x \in U$ and $y \notin U$. Since $\operatorname{Im}(e)$ is nontrivial there exist $a, b \in A$ with $a e \neq b e$. Thus, after defining $\varphi \in \mathbb{C}(X, A)$ by $U \varphi=\{a\}$ and $(X-U) \varphi=\{b\}$, we have

$$
\varphi\left(\langle x, e\rangle \mu_{X}\right)=\varphi \Gamma_{x} e=x \varphi e=a e \neq b e=y \varphi e=\varphi \Gamma_{y} e=\varphi\left(\langle y, e\rangle \mu_{X}\right) .
$$

It follows at once that $\mu_{X}$ is one-to-one.

For each $\varphi \in \mathcal{C}(X, A)$ and each $a \in A$ let

$$
(\varphi ; a)=\{g \in \mathrm{A}(\mathrm{C}(X, A), A) \mid \varphi g=a\} .
$$

Since $A$ is finite, $\{(\varphi ; a) \mid \varphi \in \mathcal{C}(X, A) ; a \in A\}$ is a subbasis for the topology on $\mathrm{A}(\mathrm{C}(X, A), A)$. Let $U=\{\langle x, e\rangle \in\{\}(X) \mid x \varphi e=a\}$. If $\{a\}$ is not a subalgebra of $A$, then $(\varphi ; a) \mu_{X}^{-1}=U$, and if $\{a\}$ is a subalgebra of $A$, then $(\varphi ; a) \mu_{X}^{-1}=$ $U \cup\{\bar{a}\}$. Hence to prove that $\mu_{X}$ is continuous it is sufficient to prove that $U$ is open in $X$; but, for every $\langle x, e\rangle \in U,\left(a e^{-1}\right) \varphi^{-1} \times\{e\}$ is an open neighbourhood of $\langle x, e\rangle$ contained in $U$. Thus $\mu_{X}$ is continuous, and, since it is a bijection, it is a homeomorphism.

Remark 3.4. Let $B$ be a Boolean algebra and let $A$ be a finite algebra. It is easily seen that the Boolean extension $A[B]$ of $A$ by $B$ (see [17]) is isomorphic to $C(X, A)$, where $X$ is the Stone space of $B$. Thus Corollary 3.3 implies that, under the assumptions of the proposition, $\mathrm{A}(A[B], A)$ is homeomorphic to $\mathfrak{F}(X)$.

For all $X \in \mathrm{ZComp} \mathrm{let} \mathcal{F}^{1}(X)=X \times\left(\operatorname{End}\left(C_{n}^{1}\right)-\{\theta\}\right) \dot{\cup}\{\theta\}$, let $\theta$ be the distinguished point of $\mathfrak{F}^{1}(X)$, and define the action of $\operatorname{End}\left(C_{n}^{1}\right)$ on $\mathfrak{F}^{1}(X)$ by

$$
\langle x, e\rangle \tilde{f}=\left\{\begin{array}{ll}
\langle x, e f\rangle & \text { if } e f \neq \theta, \\
\theta & \text { if } e f=\theta,
\end{array} \text { and } \theta \tilde{f}=\theta .\right.
$$

If $\psi \in \mathcal{C}(X, Y)$, then define $\mathfrak{F}^{1}(\psi) \in \mathbf{X}_{n}\left(\mathfrak{F}^{1}(X), \mathfrak{F}^{1}(Y)\right)$ by $\langle x, e\rangle \mathfrak{F}^{1}(\psi)=$ $\langle x \psi, e\rangle$ and $\theta\{\}^{1}(\psi)=\theta$. Clearly \{\}$^{1}: \mathrm{ZComp} \rightarrow \mathrm{X}_{n}$ is a well-defined functor.

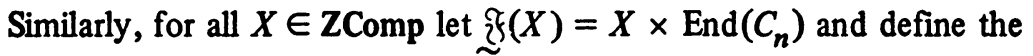
action of $\operatorname{End}\left(C_{n}\right)$ on $\mathfrak{z}(X)$ by $\langle x, e\rangle \widetilde{f}=\langle x$, ef $\rangle$. If $\psi \in \mathcal{C}(X, Y)$, then define $\mathfrak{F}(\psi) \in \mathrm{Y}_{n}(\mathfrak{F}(X), \mathfrak{F}(Y))$ by $\left.\langle x, e\rangle\right\}(\psi)=\langle x \psi, e\rangle$. Clearly $\mathfrak{F}:$ ZComp $\rightarrow$ $\mathbf{Y}_{n}$ is a well-defined functor.

Note that if $\psi \in C(X, Y)$, then $C\left(\psi, C_{n}^{1}\right): C\left(Y, C_{n}^{1}\right) \rightarrow C\left(X, C_{n}^{1}\right)$, defined 
by $\varphi \mathrm{C}\left(\psi, C_{n}^{1}\right)=\psi \varphi$, is a homomorphism, and hence $\mathrm{C}\left(-, C_{n}^{1}\right): \mathrm{ZComp}^{\mathrm{op}} \rightarrow$ $\mathrm{S}_{n}$ is a well-defined functor; the functor $C\left(-, C_{n}\right): Z_{C o m p}{ }^{o p} \rightarrow L_{n}$ is defined similarly.

THEOREM 3.5. (i) $\mathfrak{F}^{1}:$ ZComp $\rightarrow \mathbf{X}_{n}$ is naturally isomorphic to $\mathbf{L}_{n}\left(C\left(-, C_{n}^{1}\right), C_{n}^{1}\right): \mathbf{Z C o m p} \rightarrow \mathrm{X}_{n}$ and is a ZComp-free functor for $\mathbf{X}_{n}$.

(ii) $\mathfrak{F}: \mathrm{ZComp} \rightarrow \mathrm{Y}_{n}$ is naturally isomorphic to $\mathrm{S}_{n}\left(\mathrm{C}\left(-, C_{n}\right), C_{n}\right)$ : $\mathbf{Z C o m p} \rightarrow \mathrm{Y}_{n}$ and is a $\mathbf{Z C o m p - f r e e ~ f u n c t o r ~ f o r ~} \mathbf{Y}_{n}$.

Proof. We only prove (i). Since the lattice of congruences of a Brouwerian algebra $A$ is isomorphic to its lattice of filters, $A$ is subdirectly irreducible if and only if it has a unique coatom. Hence $C_{m}^{1}$ is subdirectly irreducible for all $m \geqslant 2$ and consequently $C_{n}^{1}$ satisfies the conditions of Proposition 3.2. Our first claim is that $\mu: \mathfrak{F}^{1} \rightarrow \mathrm{S}_{n}\left(\mathrm{C}\left(-, C_{n}^{1}\right), C_{n}^{1}\right)$, as defined in Corollary 3.3, is a natural isomorphism. A simple calculation shows that $\mu$ is a natural transformation, and by Corollary $3.3, \mu_{X}$ is a homeomorphism for each $X \in \mathbf{Z C o m p}$. Since, by definition, $\mu_{X}$ preserves the distinguished point, it remains only to prove that $\mu_{X}$ preserves the action of $\operatorname{End}\left(C_{n}^{1}\right)$; but again this is a simple calculation.

The unit $\zeta: \mathrm{id}_{\mathbf{Z C o m p}} \rightarrow \mathrm{X}_{n}$ of the adjunction from ZComp to $\mathbf{X}_{n}$ is defined by $x \zeta_{X}=\langle x, \imath\rangle$. It is clear that $\zeta$ is a natural transformation and we now show that $\zeta$ satisfies the universal mapping property. If $Y \in X_{n}$ and $\varphi \in C(X, Y)$, then define $\psi \in \mathrm{X}_{n}\left(\mathfrak{F}^{1}(X), Y\right)$ by $\langle x, e\rangle \psi=x \varphi e$ and $\theta \psi=1$, where $1 \mathrm{de}$ notes the distinguished point of $Y$. Since $x \zeta_{X} \psi=\langle x, \iota\rangle \psi=x \varphi$ we have $\zeta_{X} \psi$ $=\varphi$, and the uniqueness of $\psi$ is immediate.

The free functors from Set into $X_{n}$ and $Y_{n}$ may be obtained by composing $\mathfrak{F}^{1}$ and $\mathfrak{f}$ respectively with $\beta$, the Stone-Čech compactification functor.

We can now describe the sur-projectives in $X_{n}$ and $Y_{n}$; as usual, a proof is only provided for the case of $\mathrm{X}_{n}$, which is the more technical of the two.

Recall that for $1 \leqslant k<n, e_{k} \in \operatorname{End}\left(C_{n}^{1}\right)$ denotes the endomorphism determined by the prime filter $\left[c_{k}\right)$, and note that $e_{n-1}=\iota$. Let $E_{k}^{1}=e_{k} \operatorname{End}\left(C_{n}^{1}\right)$ $-\{\theta\}$ be the deleted right ideal of End $\left(C_{n}^{1}\right)$ generated by $e_{k}$. If $X_{1}, \ldots, X_{n-1}$ are (possibly empty) Boolean spaces, then

$$
Z=\dot{U}\left(X_{k} \times E_{k}^{1} \mid 1 \leqslant k<n\right) \dot{U}\{\theta\}
$$

is a subobject of $\mathfrak{F}^{1}(Y)$, where $Y=\dot{U}\left(X_{k} \mid 1 \leqslant k<n\right)$, and $\tau: \mathfrak{F}^{1}(Y) \rightarrow Z$, defined by

$$
\langle x, e\rangle \tau=\left\{\begin{array}{ll}
\left\langle x, e_{k} e\right\rangle & \text { if } x \in X_{k} \text { and } e_{k} e \neq \theta, \\
\theta & \text { if } x \in X_{k} \text { and } e_{k} e=\theta,
\end{array} \text { and } \theta \tau=\theta,\right.
$$

is a retraction onto $Z$. 
Before stating and proving the characterization of the sur-projectives in $\mathbf{X}_{\boldsymbol{n}}$ we require a lemma.

LEMMA 3.6. Let $X \in \mathrm{ZComp}$ and assume that $P$ is a subobject of $\mathfrak{F}^{1}(X)$ which is a retract. Then $\langle x, e\rangle \in P$ implies that $\left\langle x, e_{\downarrow}\right\rangle \in P$ for all $e \in$ End $\left(C_{n}^{1}\right)-\{\theta\}$

Proof. Let $\tau: \mathfrak{f}^{1}(X) \rightarrow P$ be a retraction and let $\langle x, e\rangle \in P$. If $\langle x, \iota\rangle \tau$ $=\theta$, then $\langle x, e\rangle=\langle x, e\rangle \tau=\langle x, \iota\rangle \tilde{e} \tau=\langle x, \iota\rangle \tau \tilde{e}=\theta \tilde{e}=\theta$, a contradiction; hence there exists $y \in X$ and $f \in \operatorname{End}\left(C_{n}^{1}\right)$ such that $\langle x, \imath\rangle \tau=\langle y, f\rangle$.

Now $\langle x, e\rangle=\langle x, e\rangle \tau=\langle x, \imath\rangle \tilde{e} \tau=\langle x, \iota\rangle \tau \tilde{e}=\langle y, f\rangle \tilde{e}=\langle y, f e\rangle$, and thus $x=y$ and $e=f e$. But $e=f e$ implies that $a f=a$ for all $a \in C_{n}^{1}$ for which $a e \neq 1$. Hence $f e_{\downarrow}=e_{\downarrow}$, and since $\langle x, f\rangle \in P$ it follows that $\left\langle x, e_{\downarrow}\right\rangle=\left\langle x, f e_{\downarrow}\right\rangle$ $=\langle x, f\rangle \tilde{e}_{\downarrow} \in P$.

THEOREM 3.7. The following are equivalent:

(i) $P$ is sur-projective in $\mathrm{X}_{n}$;

(ii) $P$ is a retract of $\mathfrak{F}^{1}(X)$ for some compact extremally disconnected space $X$;

(iii) there are compact extremally disconnected spaces $X_{1}, \ldots, X_{n-1}$ such that $P$ is isomorphic, in $\mathrm{X}_{n}$, to

$$
\dot{U}\left(X_{k} \times E_{k}^{1} \mid 1 \leqslant k<n\right) \dot{U}\{\theta\} .
$$

Proof. By Proposition 3.1, (i) is equivalent to (ii). If (iii) holds, then $P$ is a retract of $\mathfrak{f}^{1}\left(\dot{U}\left(X_{k} \mid 1 \leqslant k<n\right)\right)$ by the discussion above. Since each $X_{k}$ is compact and extremally disconnected so is $\dot{U}\left(X_{k} \mid 1 \leqslant k<n\right)$; hence (ii) holds. It remains only to prove that (ii) implies (iii).

Without loss of generality, assume that $P$ is a subobject of $\vartheta^{1}(X)$; let $\tau$ : $\mathcal{f}^{1}(X) \rightarrow P$ be a retraction. Let $X_{n-1}=\{x \in X \mid\langle x, \imath\rangle \in P\}$ and for $1 \leqslant k<$ $n-1$ let $X_{k}=\left\{x \in X \mid\left\langle x, e_{k}\right\rangle \in P ; x \notin X_{k+1}\right\}$. Since $e_{\downarrow} \in\left\{e_{k} \mid 1 \leqslant k<n\right\}$ for all $e \in \operatorname{End}\left(C_{n}^{1}\right)-\{\theta\}$ it follows, by Lemma 3.6, that

$$
P=\dot{U}\left(X_{k} \times E_{k}^{1} \mid 1 \leqslant k<n\right) \dot{U}\{\theta\} .
$$

A clopen subset of a retract of a compact extremally disconnected space is compact and extremally disconnected (see [16], [23]), and hence, since $\theta$ is an isolated point of $\mathfrak{q}^{1}(X)$, it is sufficient to show that $\bigcup\left(X_{k} \mid 1 \leqslant k<n\right) \dot{U}\{\theta\}$ is a retract of $X \dot{U}\{\theta\}$ and that each $X_{k}$ is clopen in $U\left(X_{k} \mid 1 \leqslant k<n\right)$.

Let $Y=\bigcup\left(X_{k} \mid 1 \leqslant k<n\right)$ and define $\sigma: X \dot{\cup}\{\theta\} \rightarrow Y \dot{U}\{\theta\}$ by $\theta \sigma=$ $\theta$ and $x \sigma=\left\langle x, e_{1}\right\rangle \tau \pi$, where $\pi: \mathfrak{F}^{1}(X) \rightarrow X \dot{\cup}\{\theta\}$ is the obvious projection. If $\langle x, e\rangle \in P$, then $\left\langle x, e_{\downarrow}\right\rangle \in P$, by Lemma 3.6, and hence $\left\langle x, e_{1}\right\rangle=\left\langle x, e_{\downarrow} e_{1}\right\rangle$ $=\left\langle x, e_{\downarrow}\right\rangle \tilde{e}_{1} \in P$. Thus for all $x \in Y, x \sigma=\left\langle x, e_{1}\right\rangle \tau \pi=\left\langle x, e_{1}\right\rangle \pi=x$. 
If $U$ is open in $X$, then

$$
\begin{aligned}
U \sigma^{-1} & =\left\{x \in X \mid\left\langle x, e_{1}\right\rangle \tau \pi \in U\right\} \\
& =\left\{x \in X \mid\left\langle x, e_{1}\right\rangle \tau \in U \times\left(\operatorname{End}\left(C_{n}^{1}\right)-\{\theta\}\right)\right\} \\
& =\left[\left(X \times\left\{e_{1}\right\}\right) \cap\left(U \times\left(\operatorname{End}\left(C_{n}^{1}\right)-\{\theta\}\right) \tau^{-1}\right] \pi,\right.
\end{aligned}
$$

which is open in $X$ since $\tau$ is continuous and $\pi$ is open. Similarly,

$$
\begin{aligned}
\{\theta\} \sigma^{-1} & =\left\{x \in X \mid\left\langle x, e_{1}\right\rangle \tau=\theta\right\} \cup\{\theta\} \\
& =\left[\left(X \times\left\{e_{1}\right\}\right) \cap\{\theta\} \tau^{-1}\right] \pi \cup\{\theta\}
\end{aligned}
$$

which is open in $X \dot{U}\{\theta\}$ since $\theta$ is an isolated point of $\mathfrak{f}^{1}(X), \tau$ is continuous, and $\pi$ is open. Hence $\sigma$ is a continuous retraction of $X \dot{U}\{\theta\}$ onto $Y \dot{U}\{\theta\}$.

To show that each $X_{k}$ is clopen in $Y$ it is sufficient to prove that for $1 \leqslant$ $k<n$ the set $U_{k}=\left\{x \in X \mid\left\langle x, e_{k}\right\rangle \in P\right\}$ is clopen in $Y$. Since $\tau$ is continuous and $\pi$ being a projection parallel to a compact factor is both open and closed, it follows that

$$
V_{k}=\left[\left(X \times\left\{e_{k}\right\}\right) \cap\left(X \times\left\{e_{k}\right\}\right) \tau^{-1}\right] \pi
$$

is clopen in $X$. We claim that $U_{k}=V_{k} \cap Y$. If $x \in U_{k}$, then $\left\langle x, e_{k}\right\rangle \in P$ and hence $x \in V_{k} \cap Y$ since $\left\langle x, e_{k}\right\rangle \tau=\left\langle x, e_{k}\right\rangle$. Conversely, assume that $x \in V_{k} \cap$ $Y$. Then $\left\langle x, e_{k}\right\rangle \tau=\left\langle y, e_{k}\right\rangle \in P$ for some $y \in X$, and there exists $l$, with $1 \leqslant$ $l<n$, such that $\left\langle x, e_{l}\right\rangle \in P$. If $l \leqslant k$, then $e_{k} e_{l}=e_{l}$, and hence $\left\langle x, e_{l}\right\rangle=$ $\left\langle x, e_{l}\right\rangle \tau=\left\langle x, e_{k}\right\rangle \tilde{e}_{l} \tau=\left\langle x, e_{k}\right\rangle \tau \tilde{e}_{l}=\left\langle y, e_{k}\right\rangle \widetilde{e}_{l}=\left\langle y, e_{l}\right\rangle$; which implies that $x=y$. Thus $\left\langle x, e_{k}\right\rangle=\left\langle y, e_{k}\right\rangle \in P$. If $\left.l\right\rangle k$, then $e_{l} e_{k}=e_{k}$, and hence $\left\langle x, e_{k}\right\rangle$ $=\left\langle x, e_{l}\right\rangle \tilde{e}_{k} \in P$ since $\left\langle x, e_{l}\right\rangle \in P$. In either case $\left\langle x, e_{k}\right\rangle \in P$, giving $x \in U_{k}$ as required.

Let $E_{k}=e_{k}$ End $\left(C_{n}\right)$ be the right ideal of $\operatorname{End}\left(C_{n}\right)$ generated by $e_{k}$. If $X_{1}, \ldots, X_{n-1}$ are (possibly empty) Boolean spaces, then

$$
Z=\dot{U}\left(X_{k} \times E_{k} \mid 1 \leqslant k<n\right)
$$

is a subobject of $\mathfrak{F}(Y)$, where $Y=\dot{U}\left(X_{k} \mid 1 \leqslant k<n\right)$, and $\tau: \mathfrak{F}(Y) \rightarrow Z$, defined by

$$
\langle x, e\rangle \tau=\left\langle x, e_{k} e\right\rangle \text { for } x \in X_{k},
$$

is a retraction onto $Z$.

THEOREM 3.8. The following are equivalent:

(i) $P$ is sur-projective in $Y_{n}$;

(ii) $P$ is a retract of $\mathfrak{F}(X)$ for some compact extremally disconnected space $X$; 
(iii) there are compact extremally disconnected spaces $X_{1}, \ldots, X_{n-1}$ such that $P$ is isomorphic, in $\mathrm{Y}_{n}$, to

$$
\dot{U}\left(X_{k} \times E_{k} \mid 1 \leqslant k<n\right) \text {. }
$$

4. Injectives and absolute subretracts in $S_{n}$ and $L_{n}$. We recall some definitions; throughout this preamble $\mathbf{A}$ denotes an equational class of universal algebras.

Definition 4.1. Let $A \in \mathbf{A} A$ is an injective in $\mathbf{A}$ if for all $B, C \in \mathbf{A}$, with $B$ a subalgebra of $C$, and every $g \in \mathrm{A}(B, A)$, there exists $g^{\prime} \in \mathrm{A}(C, A)$ with $g^{\prime} \mid B=g$. $A$ is a weak injective in $\mathbf{A}$ if for all $B, C \in \mathbf{A}$, with $B$ a subalgebra of $C$, and every surjection $g \in \mathrm{A}(B, A)$, there exists $g \in \mathrm{A}(C, A)$ with $g^{\prime} \mid B=g$. $A$ is an absolute subretract in $\mathbf{A}$ if it is a retract of each of its extensions in $\mathbf{A} . A$ is self-injective if for each subalgebra $B$ of $A$ and every $g \in \mathbf{A}(B, A)$, there exists $g^{\prime} \in \mathbf{A}(A, A)$ with $g^{\prime} \mid B=g$. A maximal subdirectly irreducible algebra in $\mathbf{A}$ is a subdirectly irreducible algebra with no subdirectly irreducible, proper extension in A. Let $\left(A_{\delta} \mid \delta \in \Delta\right)$ be a family of algebras and let $g: A \rightarrow \Pi\left(A_{\delta} \mid \delta \in \Delta\right)$ be an embedding of $A$ as a subdirect product; if $g$ also embeds $A$ as a retract of $\Pi\left(A_{\delta} \mid \delta \in \Delta\right)$, then $A$ is a subdirect retract of the family $\left(A_{\delta} \mid \delta \in \Delta\right)$. A has enough injectives if every algebra in $\mathbf{A}$ has an injective extension in $\mathbf{A}$. Finally, $\mathbf{A}$ satisfies the congruence extension property if for all $A, B \in \mathrm{A}$, with $A$ a subalgebra of $B$, and every congruence $\Theta$ on $A$, there is a congruence $\Theta^{\prime}$ on $B$ with $\Theta^{\prime} \mid A=\Theta$.

Some of the relationships which tie these concepts together are indicated in the following result (see [4], [10], [20], and [21]).

Proposition 4.2. (i) Every injective in $\mathbf{A}$ is a weak injective in $\mathbf{A}$, and every weak injective in $\mathbf{A}$ is an absolute subretract in $\mathbf{A}$.

(ii) Every maximal subdirectly irreducible algebra in $\mathbf{A}$ is an absolute subretract in $\mathbf{A}$.

(iii) A subdirect retract of a family of weak injectives in $\mathbf{A}$ is itself a weak injective in $\mathbf{A}$.

(iv) If A satisfies the congruence extension property, then $A$ is a weak injective in $\mathbf{A}$ if and only if it is an absolute subretract in $\mathbf{A}$.

(v) If A has enough injectives, then (in A) the concepts of injective, weak injective, and absolute subretract are equivalent.

(vi) Assume that every algebra in A has a distributive congruence lattice and that $\mathbf{A}=\operatorname{ISP}(\{A\})$, where $A$ is a finite, subdirectly irreducible algebra whose subalgebras are either injective or subdirectly irreducible. Then the following are equivalent: (a) A has enough injectives; (b) $A$ is injective in $\mathrm{A}$; (c) $A$ is self-injective. 
Before tackling the injectives and absolute subretracts in $S_{n}$ and $L_{n}$ we prove two further results.

If $A, B \in \mathrm{A}$ and $\Theta$ and $\Phi$ are congruences on $A$ and $B$ respectively, then define a congruence $\langle\Theta, \Phi\rangle$ on $A \times B$ by

$$
\left\langle\left\langle a_{1}, b_{1}\right\rangle,\left\langle a_{2}, b_{2}\right\rangle\right\rangle \in\langle\theta, \Phi\rangle \Longleftrightarrow\left(\left\langle a_{1}, a_{2}\right\rangle \in \Theta \text { and }\left\langle b_{1}, b_{2}\right\rangle \in \Phi\right) \text {. }
$$

If for all algebras $A, B \in \mathrm{A}$ every congruence on $A \times B$ can be factored in this manner, then $A$ has the product property on congruences. Note that if $\Psi$ is a congruence on $A \times B$ which factors as $\Psi=\langle\Theta, \Phi\rangle$, then

$$
\begin{aligned}
\left\langle a_{1}, a_{2}\right\rangle \in \Theta & \Leftrightarrow\left\langle\left\langle a_{1}, b\right\rangle,\left\langle a_{2}, b\right\rangle\right\rangle \in \Psi \text { for some } b \in B \\
& \Leftrightarrow\left\langle\left\langle a_{1}, b\right\rangle,\left\langle a_{2}, b\right\rangle\right\rangle \in \Psi \text { for all } b \in B ;
\end{aligned}
$$

the congruence $\Phi$ behaves similarly. It is well known (see [15]) that if every algebra in $\mathbf{A}$ has a distributive congruence lattice, then $\mathbf{A}$ has the product property on congruences.

LEMMA 4.3. If $\mathbf{A}$ has the product property on congruences and $\Pi\left(A_{\delta} \mid \delta \in \Delta\right)$ is an absolute subretract in $\mathbf{A}$, then each $A_{\delta}$ is also an absolute subretract in $\mathbf{A}$.

Proof. Suppose that $f: A_{\gamma} \rightarrow B$ is an embedding. Let $\Delta^{\prime}=\Delta-\{\gamma\}$ and for all $a \in \Pi\left(A_{\delta} \mid \delta \in \Delta\right)$ let $a \bar{f}=\left\langle a_{\gamma} \sigma, a^{\prime}\right\rangle$, where $a^{\prime}$ is the restriction of $a$ to $\Pi\left(A_{\delta} \mid \delta \in \Delta^{\prime}\right)$; clearly

$$
\bar{f}: \prod\left(A_{\delta} \mid \delta \in \Delta\right) \rightarrow B \times \prod\left(A_{\delta} \mid \delta \in \Delta^{\prime}\right)
$$

is an embedding. Let

$$
g: B \times \prod\left(A_{\delta} \mid \delta \in \Delta^{\prime}\right) \rightarrow \prod\left(A_{\delta} \mid \delta \in \Delta\right)
$$

be a retraction of $\bar{f}$. Let $c \in \Pi\left(A_{\delta} \mid \delta \in \Delta^{\prime}\right)$ and define $h: B \rightarrow A_{\gamma}$ by $b h=$ $\langle b, c\rangle g \pi_{\gamma}$, where $\pi_{\gamma}: \Pi\left(A_{\delta} \mid \delta \in \Delta\right) \rightarrow A_{\gamma}$ is the natural projection.

We claim that $h$ is independent of the choice of $c$. Since $A$ has the product property on congruences there exist congruences $\Theta$ and $\Phi$ on $B$ and $\Pi\left(A_{\delta} \mid \delta \in \Delta^{\prime}\right)$ respectively such that $\operatorname{Ker}\left(g \pi_{\gamma}\right)=\langle\Theta, \Phi\rangle$. Clearly it is sufficient to prove that for all $c, d \in \Pi\left(A_{\delta} \mid \delta \in \Delta^{\prime}\right),\langle c, d\rangle \in \Phi$, that is, there exists $b \in B$ such that $\langle b, c\rangle g \pi_{\gamma}=\langle b, d\rangle g \pi_{\gamma}$. Let $a \in A_{\gamma}$; then $b=a f$ will suffice since $\langle b, c\rangle g \pi_{\boldsymbol{\gamma}}=\langle a f, c\rangle g \pi_{\boldsymbol{\gamma}}=\langle a, c\rangle \bar{f} g \pi_{\boldsymbol{\gamma}}=\langle a, c\rangle \pi_{\boldsymbol{\gamma}}=a$, and similarly, $\langle b, d\rangle g \pi_{\boldsymbol{\gamma}}$ $=a$.

It follows immediately that $h$ is a homomorphism, and, since $f h=\mathrm{id}_{A \gamma}$, we are through.

The following result was proved in [21] for the case in which $\mathbf{A}$ is an equational class of distributive pseudocomplemented lattices. 
Proposition 4.4. If $A$ is a finite, weak injective algebra in $\mathrm{A}$, then $\mathrm{C}(X, A)$ is a weak injective in A for every compact extremally disconnected space $X$.

PROof. It is readily verified that the functor $\mathrm{C}(-, A): \mathbf{Z C o m p}^{\mathrm{OP}} \rightarrow \mathbf{A}$ has the following properties: (a) If $\varphi$ is onto, then $\mathrm{C}(\varphi, A)$ is one-to-one; (b) if $\varphi$ is one-to-one, then $\mathrm{C}(\varphi, A)$ is onto; (c) if $\left(X_{\delta} \mid \delta \in \Delta\right)$ is a family of Boolean spaces and $X=\beta\left(\dot{U}\left(X_{\delta} \mid \delta \in \Delta\right)\right)$ is their coproduct in ZComp, then $C(X, A) \cong$ $\Pi\left(\mathrm{C}\left(X_{\delta}, A\right) \mid \delta \in \Delta\right)$. Indeed, (a) is trivial, (b) follows from the fact that every finite space is injective in ZComp, and (c) follows from well-known properties of the Stone-Čech compactification.

For every $X \in Z$ Comp there is a surjection $\psi \in C(\beta S, X)$ for some discrete space $S$ (e.g. let $S$ be the underlying set of $X$ ), from which it follows, by (a), (b), and (c), that $C(\psi, A)$ is an embedding of $C(X, A)$ into $C(\beta S, A) \cong A^{S}$ as a subdirect product. Since $X$ is extremally disconnected it is sur-projective in ZComp (see [16] or [23]) and hence $\psi: \beta S \rightarrow X$ is a retraction. Since any functor preserves retractions it follows that $\mathrm{C}(\psi, A)$ embeds $\mathrm{C}(X, A)$ in $\mathrm{C}(\beta S, A)$ as a retract, whence $C(X, A)$ is a subdirect retract of copies of $A$. Hence $C(X, A)$ is a weak injective in A by Proposition 4.2(iii).

REMARK 4.5. Since compact extremally disconnected spaces are precisely the Stone spaces of complete Boolean algebras (see [23]), Proposition 4.4 may be restated as follows (cf. Remark 3.4).

If $A$ is a finite, weak injective algebra in $\mathbf{A}$, then for every complete Boolean algebra $B$ the Boolean extension $A[B]$ of $A$ by $B$ is a weak injective in $\mathbf{A}$.'

We now have more than enough machinery to handle the injectives and absolute subretracts in $S_{n}$ and $L_{n}$.

Lemma 4.6. Let $3 \leqslant n<\omega$. Then for any nonempty Boolean space $X$, $C\left(X, C_{n}^{1}\right)$ is not self-injective, and for $2 \leqslant k<n, C\left(X, C_{k}^{1}\right)$ is not an absolute subretract in $\mathbf{S}_{n}$.

Proof. Let $A=\left\{c_{n-2}, 1\right\} \subset C_{n}^{1}$. Then $C(X, A)$ is a subalgebra of $C\left(X, C_{n}^{1}\right)$. Define $g: A \rightarrow C_{n}^{1}$ by $c_{n-2} g=0$ and $1 g=1$, and define $\bar{g}$ : $\mathrm{C}(X, A) \rightarrow \mathrm{C}\left(X, C_{n}^{1}\right)$ by $\varphi \bar{g}=\varphi g$. If $\mathrm{C}\left(X, C_{n}^{1}\right)$ were self-injective there would be a homomorphism $h: C\left(X, C_{n}^{1}\right) \rightarrow C\left(X, C_{n}^{1}\right)$ satisfying $\varphi h=\varphi \bar{g}$ for all $\varphi \in$ $C(X, A)$. For each $c \in C_{n}^{1}$ let $\hat{c} \in C\left(X, C_{n}^{1}\right)$ be the corresponding constant map. Since $\hat{0}=\hat{c}_{n-2} \bar{g}=\hat{c}_{n-2} h$ and $\hat{c}_{n-2} * \hat{0}=\hat{0}$ we obtain

$$
\hat{0} * \hat{0} h=\hat{c}_{n-2} h * \hat{0} h=\left(\hat{c}_{n-2} * \hat{0}\right) h=\hat{0} h \text {, }
$$

which gives the contradiction $\hat{0}>\hat{0} h$. Hence $C\left(X, C_{n}^{1}\right)$ is not self-injective. Now let $g: C_{k}^{1} \rightarrow C_{n}^{1}$ embed $C_{k}^{1}$ as a filter of $C_{n}^{1}$, and define $\bar{g}: C\left(X, C_{k}^{1}\right)$ 
$\rightarrow C\left(X, C_{n}^{1}\right)$ by $\varphi \bar{g}=\varphi g$. If $C\left(X, C_{k}^{1}\right)$ is an absolute subretract, then there exists a retraction $h: \mathcal{C}\left(X, C_{n}^{1}\right) \rightarrow C\left(X, C_{k}^{1}\right)$ of $\bar{g}$. Since $0 g>0$ it follows that $\hat{0} \bar{g} * \hat{0}=\hat{0}$, and hence

$$
\hat{0} * \hat{0} h=\hat{0} \bar{g} h * \hat{0} h=(\hat{0} \bar{g} * \hat{0}) h=\hat{0} h ;
$$

again we have the contradiction $\hat{0}>\hat{0} h$. Hence $C\left(X, C_{k}^{1}\right)$ is not an absolute subretract in $S_{n}$.

If $B$ is a Boolean algebra, then let

$$
\mathrm{n}[B]=\left\{\left\langle b_{0}, \ldots, b_{n-2}\right\rangle \in B^{n-1} \mid b_{0} \leqslant b_{1} \leqslant \cdots \leqslant b_{n-2}\right\} .
$$

It is easily seen that $\mathbf{n}[B]$ is a Brouwerian (in fact, Heyting) algebra in which

$$
\begin{aligned}
& \left\langle a_{0}, \ldots, a_{n-2}\right\rangle *\left\langle b_{0}, \ldots, b_{n-2}\right\rangle \\
& =\left\langle\bigwedge_{i=0}^{n-2} a_{i}^{\prime} \vee b_{i}, \bigwedge_{i=1}^{n-2} a_{i}^{\prime} \vee b_{i}, \ldots, a_{n-2}^{\prime} \vee b_{n-2}\right\rangle .
\end{aligned}
$$

Furthermore, it is readily verified that $\mathrm{n}[B] \cong C\left(X, C_{n}^{1}\right)$, where $X$ is the Stone space of $B$, and hence $\mathbf{n}[B] \in \mathbf{S}_{n}$.

For the definition and a discussion of $n$-valued Post algebras we refer to G. Epstein [14]. Only the following facts are required here: (a) for every Boolean algebra $B, \mathrm{n}[B]$ is an $n$-valued Post algebra, and conversely, each $n$-valued Post algebra $A$ is isomorphic to $\mathrm{n}[B]$, where $B$ is the centre of $A$ (the centre of a bounded distributive lattice is its Boolean algebra of complemented elements); (b) an $n$-valued Post algebra is complete if and only if its centre is complete. For convenience, we regard the one-element algebra as an $n$-valued Post algebra.

THEOREM 4.7. The following are equivalent:

(i) $A$ is a weak injective in $\mathrm{S}_{n}$;

(ii) $A$ is an absolute subretract in $\mathbf{S}_{n}$;

(iii) there is a compact extremally disconnected space $X$ such that $A \cong$ $C\left(X, C_{n}^{1}\right)$;

(iv) there is a complete Boolean algebra $B$ such that $A \cong \mathrm{n}[B]$;

(v) $A$ is a complete n-valued Post algebra.

Proof. (i) $\Longleftrightarrow$ (ii). This equivalence follows from Proposition 4.2(iv) since Brouwerian algebras have the congruence extension property.

(ii) $\Longleftrightarrow$ (iii). As we noted in the proof of Theorem 2.4, the set $S=$ $\mathrm{S}_{n}\left(A, C_{n}^{1}\right)$ separates the points of $A$. Thus $A$ is isomorphic to a subalgebra of $\left(C_{n}^{1}\right)^{S} \cong C\left(\beta S, C_{n}^{1}\right)$, and so $A$ is a retract of $C\left(\beta S, C_{n}^{1}\right)$. It follows that $S_{n}\left(A, C_{n}^{1}\right)$ 
is a retract of $\mathrm{S}_{n}\left(\mathrm{C}\left(\beta S, C_{n}^{1}\right), C_{n}^{1}\right)$, which, by Theorem 3.5, is isomorphic (in $\mathrm{X}_{n}$ ) to $\mathcal{F}^{1}(\beta S)$. Since $\beta S$ is extremally disconnected, Theorem 3.7 implies that there are (possibly empty) compact extremally disconnected spaces $X_{1}, \ldots, X_{n-1}$ such that $\mathrm{S}_{n}\left(A, C_{n}^{1}\right)$ is isomorphic to

$$
Z=\dot{U}\left(X_{k} \times E_{k}^{1} \mid 1 \leqslant k<n\right) \dot{U}\{\theta\} .
$$

For $1 \leqslant k<n, Z_{k}=\left(X_{k} \times E_{k}^{1}\right) \dot{\cup}\{\theta\}$ is a subobject of $Z$, and if $\varphi_{k} \in$ $\mathbf{X}_{n}\left(Z_{k}, C_{n}^{1}\right)$, then $\varphi \in \mathbf{X}_{n}\left(Z, C_{n}^{1}\right)$ may be defined by $\varphi \mid Z_{k}=\varphi_{k}$; in fact, $Z$ is the $\mathrm{X}_{n}$-coproduct of the family $\left(Z_{k} \mid 1 \leqslant k<n\right)$. Hence, by Theorem 2.5 ,

$$
\begin{aligned}
A & \cong \mathrm{X}_{n}\left(\mathrm{~S}_{n}\left(A, C_{n}^{1}\right), C_{n}^{1}\right) \cong \mathrm{X}_{n}\left(Z, C_{n}^{1}\right) \\
& \cong \prod\left(\mathrm{X}_{n}\left(Z_{k}, C_{n}^{1}\right) \mid 1 \leqslant k<n\right) .
\end{aligned}
$$

We claim that $\mathrm{X}_{n}\left(Z_{k}, C_{n}^{1}\right) \cong \mathrm{C}\left(X_{k}, C_{k+1}^{1}\right)$. If $\varphi \in \mathrm{X}_{n}\left(Z_{k}, C_{n}^{1}\right)$, then

$$
\left\langle x, e_{k}\right\rangle \varphi=\left\langle x, e_{k}\right\rangle \tilde{e}_{k} \varphi=\left\langle x, e_{k}\right\rangle \varphi e_{k},
$$

and thus $\left\langle x, e_{k}\right\rangle \varphi \in \operatorname{Im}\left(e_{k}\right)=\left\{0, c_{1}, \ldots, c_{k-1}, 1\right\}$. Hence $\varphi$ induces a map $\hat{\varphi} \in \mathcal{C}\left(X_{k}, C_{k+1}^{1}\right)$ defined by $x \hat{\varphi}=\left\langle x, e_{k}\right\rangle \varphi$. Conversely, each map $\psi \in$ $C\left(X_{k}, C_{k+1}^{1}\right)$ induces a map $\hat{\psi} \in \mathbf{X}_{n}\left(Z_{k}, C_{n}^{1}\right)$ defined by $\langle x, e\rangle \hat{\psi}=x \psi e$ and $\theta \hat{\psi}=\theta$. The map $\varphi \rightarrow \hat{\varphi}$ is clearly a homomorphism and since $\hat{\varphi}=\varphi$ and $\hat{\hat{\psi}}=\psi$ it follows that $\mathbf{X}_{n}\left(Z_{k}, C_{n}^{1}\right) \cong C\left(X_{k}, C_{k+1}^{1}\right)$.

Thus $A \cong \Pi\left(C\left(X_{k}, C_{k+1}^{1}\right) \mid 1 \leqslant k<n\right)$; but, since $A$ is an absolute subretract, Lemma 4.3 and Lemma 4.6 imply that $X_{k}$ is empty for $1 \leqslant k<n-1$, and hence (iii) follows.

(iii) $\Rightarrow$ (i). Since Brouwerian algebras have the congruence extension property and since $C_{n}^{1}$ is a maximal subdirectly irreducible algebra in $S_{n}$ it follows, by Proposition 4.2(ii) (iv), that $C_{n}^{1}$ is a weak injective in $S_{n}$. Hence (iii) implies (i) by Proposition 4.4.

(iii) $\Longleftrightarrow$ (iv) $\Longleftrightarrow$ (v). Since a Boolean algebra is complete if and only if its Stone space is extremally disconnected these equivalences follow from the discussion preceding the statement of the theorem.

THEOREM 4.8. $\mathrm{S}_{n}$ has enough injectives if and only if $n=2$. An algebra in $\mathrm{S}_{2}$ is injective if and only if it is a complete Boolean algebra. And for $3 \leqslant n<\omega$, $\mathrm{S}_{n}$ has only trivial injectives.

Proof. $C_{2}^{1}$ is trivially self-injective and by Lemma 4.6 , with $|X|=1, C_{n}^{1}$ is not self-injective for all $n \geqslant 3$. Hence, by Proposition 4.2(vi), only $S_{2}$ has enough injectives. By Proposition 4.2(v) and Theorem 4.7 an algebra in $S_{2}$ is injective if and only if it is a complete Boolean algebra since a 2-valued Post algebra is nothing more than a Boolean algebra. Since injective algebras are both 
self-injective and weak injective, Lemma 4.6 and Theorem 4.7 imply that for $n \geqslant 3, S_{n}$ has only trivial injectives.

We turn now to $L_{n}$. Since the proofs are very similar to the corresponding proofs for $S_{n}$ they will only be sketched.

LEMma 4.9. Let $4 \leqslant n<\omega$. Then for any nonempty Boolean space $X$, $\mathrm{C}\left(X, C_{n}\right)$ is not self-injective, and for $3 \leqslant k<n, C\left(X, C_{k}\right)$ is not an absolute subretract in $\mathbf{L}_{n}$.

Proof. Mimic the proof of Lemma 4.6. Assume that $C\left(X, C_{n}\right)$ is selfinjective and let $A=\left\{0, c_{n-2}, 1\right\}$. Define $g: A \rightarrow C_{n}$ by $0 g=0, c_{n-2} g=c_{1}$, and $1 g=1$, define $\bar{g}: C(X, A) \rightarrow C\left(X, C_{n}\right)$ by $\varphi \bar{g}=\varphi g$, and let $h$ be an extension of $\bar{g}$ to an endomorphism of $C\left(X, C_{n}\right)$. We find that $\hat{c}_{1} * \hat{c}_{1} h=\hat{c}_{1} h$, and so $\hat{c}_{1} h=\hat{0}$, giving the contradiction

$$
\hat{0}=\hat{0} h=\left(\hat{c}_{1} * \hat{0}\right) h=\hat{c}_{1} h * \hat{0} h=\hat{0} * \hat{0}=\hat{1}
$$

Assume that $\mathrm{C}\left(X, C_{k}^{1}\right)$ is an absolute subretract in $\mathrm{L}_{n}$, and let $g: C_{k} \rightarrow C_{n}$ be the embedding characterized by $0 g=0$ and $\left[c_{1}\right) g$ is a filter of $C_{n}$. Define $\bar{g}: C\left(X, C_{k}\right) \rightarrow C\left(X, C_{n}\right)$ by $\varphi \bar{g}=\varphi g$, and let $h$ be a retraction of $\bar{g}$. Again we find that $\hat{c}_{1} * \hat{c}_{1} h=\hat{c}_{1} h$, and so $\hat{c}_{1} h=\hat{0}$. This gives rise to the contradiction $\hat{0}=\hat{1}$, as above.

THEOREM 4.10. The following are equivalent:

(i) $A$ is a weak injective in $\mathrm{L}_{n}$;

(ii) $A$ is an absolute subretract in $\mathrm{L}_{n}$;

(iii) there are compact extremally disconnected spaces $X_{0}$ and $X_{1}$ such that $A \cong \mathrm{C}\left(X_{0}, C_{2}\right) \times \mathrm{C}\left(X_{1}, C_{n}\right)$ $\mathbf{n}\left[B_{1}\right]$

(iv) there are complete Boolean algebras $B_{0}$ and $B_{1}$ such that $A \cong B_{0} \times$

(v) there is a complete Boolean algebra $B$ and a complete n-valued Post algebra $P$ such that $A \cong B \times P$.

Proof. A proof can be obtained by making the obvious changes in the proof of Theorem 4.7. In particular, note that for any compact extremally disconnected space $X, C\left(X, C_{2}\right)$ is isomorphic to the complete Boolean algebra of clopen subsets of $X$, and hence $C\left(X, C_{2}\right)$ is an injective in $L_{n}$ since every complete Boolean algebra is an injective Heyting algebra (see [3]). Cearly, where Lemma 4.6 was applied in the proof of Theorem 4.7 we now call on Lemma 4.9.

Except for the characterization of the injectives in $\mathbf{L}_{3}$, the following result is due to A. Day [12].

THEOREM 4.11. $\mathbf{L}_{n}$ has enough injectives if and only if $n=2$ or $n=3$. An 
algebra in $\mathbf{L}_{2}$ is injective if and only if it is a complete Boolean algebra. An algebra in $\mathbf{L}_{3}$ is injective if and only if it is isomorphic to the direct product of a complete Boolean algebra with a complete 3-valued Post algebra. For $4 \leqslant n<\omega$, an algebra in $\mathbf{L}_{n}$ is injective if and only if it is a complete Boolean algebra.

Proof. Only the characterization of the injectives in $L_{n}$ for $n \geqslant 4$ requires more than a direct translation of the proof of Theorem 4.8. As was noted in the proof of the previous theorem, complete Boolean algebras are injective in $\mathbf{L}_{n}$. If $A$ is injective in $\mathrm{L}_{n}, n \geqslant 4$, then it is a weak injective in $\mathrm{L}_{n}$ and hence there are Boolean algebras $B_{0}$ and $B_{1}$ such that $A \cong B_{0} \times \mathrm{n}\left[B_{1}\right]$. We shall show that if $B_{1}$ is nontrivial, then $\mathrm{n}\left[B_{1}\right]$ is a retract of $B_{0} \times \mathrm{n}\left[B_{1}\right]$. Since a retract of an injective algebra is injective this contradicts the fact that $\mathrm{n}\left[B_{1}\right]$ is not self-injective.

Let $F$ be a maximal filter of $\mathrm{n}\left[B_{1}\right]$ and define $g: \mathrm{n}\left[B_{1}\right] \rightarrow B_{0} \times \mathrm{n}\left[B_{1}\right]$ by

$$
a g= \begin{cases}\langle 1, a\rangle & \text { if } a \in F, \\ \langle 0, a\rangle & \text { if } a \notin F .\end{cases}
$$

By Proposition 1.1(iii), $g$ is a homomorphism, and it is clear that the natural projection of $B_{0} \times \mathrm{n}\left[B_{1}\right]$ onto $\mathrm{n}\left[B_{1}\right]$ is a retraction of $g$.

REMARK 4.12. Recently, T. Katriňák and A. Mitschke [30] have characterized Post algebras, and R. Beazer [5] has characterized algebras of the form $B_{0} \times$ $\mathbf{n}\left[B_{1}\right]$, in terms of their Brouwerian algebra structure. These characterizations may be used to give algebraic proofs of Theorem 4.7 and Theorem 4.10 along the lines of the proof of Theorem 2 in G. Grätzer and H. Lakser [21].

5. Free products and free algebras in $S_{n}$ and $L_{n}$. Free products in $S_{n}$ and $\mathbf{L}_{n}$ are readily described via the dualities. The free product of the family $\left(A_{\delta} \mid \delta \in \Delta\right)$ is denoted by ${ }^{*} \Pi\left(A_{\delta} \mid \delta \in \Delta\right)$.

THEOREM 5.1. (i) Let $\left(A_{\delta} \mid \delta \in \Delta\right)$ be a family of algebras of $\mathrm{S}_{n}$ and let $X_{\delta}=\mathrm{S}_{n}\left(A_{\delta}, C_{n}^{1}\right)$. Then

$$
{ }^{*} \prod\left(A_{\delta} \mid \delta \in \Delta\right) \cong \mathrm{X}_{n}\left(\Pi\left(X_{\delta} \mid \delta \in \Delta\right), C_{n}^{1}\right) .
$$

(ii) Let $\left(A_{\delta} \mid \delta \in \Delta\right)$ be a family of nontrivial algebras of $\mathbf{L}_{n}$ and let $X_{\delta}=$ $\mathrm{L}_{n}\left(A_{\delta}, C_{n}\right)$. Then

$$
{ }^{*} \prod\left(A_{\delta} \mid \delta \in \Delta\right) \cong \mathrm{Y}_{n}\left(\prod\left(X_{\delta} \mid \delta \in \Delta\right), C_{n}\right)
$$

Proof. We only prove (i). Let $\mathbf{X}_{n}^{\prime}$ be the image, under the functor $S_{n}\left(-, C_{n}^{1}\right)$, of $S_{n}$. Since $S_{n}$ is equivalent to the dual of $X_{n}^{\prime}$ it follows that the image, under the functor $\mathbf{X}_{n}\left(-, C_{n}^{1}\right)$, of a direct product in $\mathbf{X}_{n}^{\prime}$ is a coproduct in $\mathrm{S}_{n}$. Free products are only distinguished from coproducts by the requirement that the natural homomorphism $g_{\gamma}: A_{\gamma} \rightarrow{ }^{*} \Pi\left(A_{\delta} \mid \delta \in \Delta\right)$ be an embedding. But 
$g_{\gamma}=\eta_{A} \mathbf{X}_{n}\left(\pi_{\gamma}, C_{n}^{1}\right)$, where $\pi_{\gamma}: \Pi\left(X_{\delta} \mid \delta \in \Delta\right) \rightarrow X_{\gamma}$ is the natural projection, and thus $g_{\gamma}$ is an embedding since $\pi_{\gamma}$ is a surjection.

In 5.1(ii) the algebra $A_{\delta}$ is assumed to be nontrivial so that $X_{\delta}$ will be nonempty; the free product of $C_{1}$ and $C_{2}$ does not exist in $\mathbf{L}_{n}$ and hence this requirement is necessary.

For all $A \in \mathrm{S}_{n}$ let $\mathrm{P}_{n}^{1}(A)$ be the subset of $\mathrm{S}_{n}\left(A, C_{n}^{1}\right)$ consisting of those homomorphisms which are determined by some prime filter of $A$ (see Definition 1.2). If $P_{n}^{1}(A)$ is ordered pointwise, then the correspondence $F \rightarrow g_{F}$ is an order-isomorphism between the poset $\mathfrak{X}(A)$ of prime filters of $A$ and the poset $P_{n}^{1}(A)$. Observe that if $g, h \in P_{n}^{1}(A)$, then

$$
g \leqslant h \text { if and only if } g e=h \text { for some } e \in \operatorname{End}\left(C_{n}^{1}\right) \text {. }
$$

Since each finite distributive lattice is determined by its poset of prime filters (see Remark 2.3) it follows that every finite algebra $A \in \mathrm{S}_{n}$ is determined by the poset $\mathrm{P}_{n}^{1}(A)$. For an algebra $A \in \mathrm{L}_{n}, \mathrm{P}_{n}(A)$ is defined similarly, and again, if $A$ is finite, then the poset $P_{n}(A)$ determines $A$.

Using this observation we can completely describe the finitely generated free algebras in $S_{n}$ and $L_{n}$. For any equational class $A$ and any ordinal $k$ let $\mathfrak{F}_{\mathrm{A}}(\kappa)$ denote the $\kappa$-generated free algebra in $A$ with free generators $\left\{x_{\gamma} \mid \gamma<\kappa\right\}$.

Define the action of End $\left(C_{n}^{1}\right)$ on $\left(C_{n}^{1}\right)^{k}$ pointwise; then it is clear that the map $\rho_{\kappa}: S_{n}\left(\mho_{S_{n}}(\kappa), C_{n}^{1}\right) \rightarrow\left(C_{n}^{1}\right)^{\kappa}$, defined by $g \rho_{\kappa}=\left\langle x_{\gamma} g\right\rangle_{\gamma<\kappa}$, is an isomorphism in $X_{n}$. Let $P_{n}^{1}(k)$ be the image of $P_{n}^{1}\left(\mho_{S_{n}}(k)\right)$ under $\rho_{k}$ and define a partial order on $P_{n}^{1}(K)$ by

$$
a \leqslant b \Longleftrightarrow\left(a \tilde{e}=b \text { for some } e \in \operatorname{End}\left(C_{n}^{1}\right)\right) \text {. }
$$

Clearly $P_{n}^{1}\left(\mathfrak{F}_{S_{n}}(\kappa)\right)$ and $P_{n}^{1}(\kappa)$ are order-isomorphic.

Proposition 5.2. (i) $\mathfrak{F}_{\mathrm{S}_{n}}(\kappa) \cong \mathrm{X}_{n}\left(\left(C_{n}^{1}\right)^{\kappa}, C_{n}^{1}\right)$.

(ii) Let $a \in\left(C_{n}^{1}\right)^{\kappa}$. Then $a \in P_{n}^{1}(\kappa)$ if and only if there exists $l$, with $1 \leqslant$ $l<n$, such that

$$
\left\{a_{\gamma} \mid \gamma<k\right\} \cup\{1\}=\left(c_{l-1}\right] \cup\{1\}
$$

Proof. (i) Apply the duality.

(ii) If $g \in \mathrm{S}_{n}\left(\mathcal{F}_{\mathrm{S}_{n}}(\kappa), C_{n}^{1}\right)$, then $\operatorname{Im}(g)$ is the subalgebra of $C_{n}^{1}$ generated by $\left\{x_{\gamma} g \mid \gamma<\kappa\right\}$, and hence $\operatorname{Im}(g)=\left\{x_{\gamma} g \mid \gamma<\kappa\right\} \cup\{1\}$, since for all $c, d \in$ $C_{n}^{1}, c * d \in\{c, d, 1\}$. Thus $g$ is determined by a prime filter of $\mathfrak{F}_{\mathrm{s}_{n}}(\kappa)$ if and only if $\left\{x_{\gamma} g \mid \gamma<\kappa\right\} \cup\{1\}=\left(c_{l-1}\right] \cup\{\rho\}$, where $1 \leqslant l<n$. After translating from $S_{n}\left(\mathfrak{F}_{S_{n}}(\kappa), C_{n}^{1}\right)$ to $\left(C_{n}^{1}\right)^{\kappa}$ via $\rho_{\kappa}$ the result follows. 
Recall that ${ }_{0} A$ is obtained from $A$ by adjoining a new zero.

THEOREM 5.3. (i) $\mathfrak{F}_{S_{2}}(m) \cong\left(C_{2}^{1}\right)^{2^{m}-1}$ for all $m<\omega$.

(ii) For $n \geqslant 3, \xi_{s_{n}}(0) \cong C_{1}^{1}$ and for $1 \leqslant m<\omega$,

$$
\mathfrak{F}_{\mathrm{s}_{n}}(m) \cong \prod_{k=0}^{m-1}\left[{ }_{0}\left(\xi_{\mathrm{s}_{n-1}}(k)\right)\right]^{\left(\begin{array}{c}
m \\
k
\end{array}\right)} \text {. }
$$

Proof. It is clear that $\xi_{S_{n}}(0) \cong C_{1}^{1}$ for all $n \geqslant 2$, and hence we assume that $m \geqslant 1$.

By applying Proposition 5.1(i) we find that $\mathfrak{F}_{\mathrm{S}_{2}}(m) \cong\left(C_{2}^{1}\right)^{2^{m}-1}$ since $\mathrm{X}_{2}\left(\left(C_{2}^{1}\right)^{m}, C_{2}^{1}\right)$ is the set of all morphisms which map $\langle 1, \ldots, 1\rangle$ to 1 and map each of the other $2^{m}-1$ elements of $\left(C_{2}^{1}\right)^{m}$ arbitrarily into $C_{2}^{1}$.

Now consider $n \geqslant 3$. If $a \in P_{n}^{1}(m)$, then, by Proposition 5.2(ii), there exists $i<m$ such that $c_{i}=0$. Thus if $a, b \in P_{n}^{1}(m)$ and $a \leqslant b$, then $a_{i}=0 \Longleftrightarrow$ $b_{i}=0$ (if $a \tilde{e}=b$, then $0 e=0$; for otherwise $a \tilde{e} \notin P_{n}^{1}(m)$ ). Hence $M^{1}=$ $\left(C_{2}^{1}\right)^{m}-\{\langle 1, \ldots, 1\rangle\}$ is the set of maximal elements of $P_{n}^{1}(m)$, and, in fact, $P_{n}^{1}(m)$ is the disjoint union of the family $\left((a] \mid a \in M^{1}\right)$. By Remark 2.3, $\mathfrak{F}_{\mathrm{S}_{n}}(m)$ is isomorphic to the lattice of increasing subsets of $P_{n}^{1}(m)$ and so is isomorphic to $\Pi\left(\mathscr{Q}((a]) \mid a \in M^{1}\right)$, where $\mathscr{U}((a])$ is the lattice of increasing subsets of $(a]$. It is easily verified that if $a \in M^{1}$ has exactly $k<m$ coordinates equal to 1 (and hence $m-k$ equal to 0 ), then (a] is order-isomorphic to $P_{n-1}^{1}(k) \cup\{\langle 1, \ldots, 1\rangle\}$. Since there are $\left(\begin{array}{c}m \\ k\end{array}\right)$ elements of $M^{1}$ with exactly $k$ coordinates equal to 1 and since the lattice of increasing subsets of $P_{n-1}^{1}(k) \cup\{\langle 1, \ldots, 1\rangle\}$ is isomorphic to ${ }_{0}\left(\gtrless_{s_{n-1}}(k)\right)$ the result follows.

The action of $\operatorname{End}\left(C_{n}\right)$ on $\left(C_{n}\right)^{K}$ is defined pointwise. The isomorphism $\rho_{\kappa}$ and the poset $P_{n}(\kappa)$ are defined for the category $Y_{n}$ as they were for $X_{n}$. As before, the partial order on $P_{n}(\kappa)$ is defined by

$$
a \leqslant b \Longleftrightarrow\left(a \tilde{e}=b \text { for some } e \in \operatorname{End}\left(C_{n}\right)\right) \text {. }
$$

The proof of the following result is similar to the proof of Proposition 5.2 and is omitted.

Proposition 5.4. (i) $\mathfrak{F}_{\mathrm{L}_{n}}(\kappa) \cong \mathrm{Y}_{n}\left(\left(C_{n}\right)^{\kappa}, C_{n}\right)$.

(ii) Let $a \in\left(C_{n}\right)^{\kappa}$. Then $a \in P_{n}(\kappa)$ if and only if there exists $l$, with $0 \leqslant$ $l<n$, such that

$$
\{0\} \cup\left\{a_{\gamma} \mid \gamma<_{k}\right\} \cup\{1\}=\{0\} \cup\left[c_{1}, c_{l-1}\right] \cup\{1\} .
$$

THEOREM 5.5. (i) $\mathfrak{F}_{\mathbf{L}_{2}}(m) \cong\left(C_{2}\right)^{2^{m}}$ for all $m<\omega$.

(ii) For $n \geqslant 3$ and $m<\omega$, 


$$
\mathfrak{F}_{\mathrm{L}_{n}}(m) \cong \prod_{k=0}^{m}\left[{ }_{0}\left(\mho_{\mathrm{S}_{n-1}}(k)\right)\right]^{\left(\begin{array}{c}
m \\
k
\end{array}\right)} \text {. }
$$

Proof. Again it is clear that $\mathfrak{F}_{\mathbf{L}_{n}}(0) \cong C_{2}$ for all $n \geqslant 2$, and hence we assume that $m \geqslant 1$.

That $\mathfrak{F}_{\mathrm{L}_{2}}(m) \cong\left(C_{2}\right)^{2^{m}}$ follows immediately from Proposition 5.4(i) since $C_{2}$ has no proper endomorphisms.

Now consider $n \geqslant 3$. It follows as in the proof of Theorem 5.3 that $M=$ $\left(C_{2}\right)^{m}$ is the set of maximal elements in the poset $P_{n}(m)$ and that $P_{n}(m)$ is the disjoint union of the family $((a] \mid a \in M)$. Consequently $\mathfrak{F}_{\mathbf{L}_{n}}(m)$ is isomorphic to $\Pi(\mathfrak{Z}((a]) \mid a \in M)$, where $\mathcal{U}((a])$ is the lattice of increasing subsets of $(a]$. Now if $a \in M$ has exactly $k$ coordinates equal to 1 , then $(a]$ is order-isomorphic to $P_{n-1}^{1}(k) \cup\{\langle 1, \ldots, 1\rangle\}$ since

(a) if $a, b \in P_{n}(m)$ and $a \leqslant b$, then $a_{i}=c_{1} \Longleftrightarrow b_{i}=c_{1}$, and

(b) by identifying $C_{n-1}^{1}$ with the filter $\left[c_{1}\right)$ of $C_{n}$ we find that $\operatorname{End}\left(C_{n}\right)$ $\cong \operatorname{End}\left(C_{n-1}^{1}\right)$.

Since there are $\left(\begin{array}{c}m \\ k\end{array}\right)$ elements of $M$ with exactly $k$ coordinates equal to 1 and since the lattice of increasing subsets of $P_{n-1}^{1}(k) \cup\{\langle 1, \ldots, 1\rangle\}$ is isomorphic to ${ }_{0}\left(\mathfrak{F}_{\mathrm{s}_{n-1}}(k)\right)$ the result follows.

The following simple result allows us to relate the free algebras in the various classes and also enables us to describe the finitely generated free algebras in $\mathbf{S}_{\boldsymbol{\omega}}$ and $\mathbf{L}_{\boldsymbol{\omega}}$.

Lemma 5.6. (i) Let $A \in \mathrm{S}_{\omega}$. If $A$ is n-generated then $A \in \mathrm{S}_{n+1}$.

(ii) Let $A \in \mathbf{L}_{\omega}$. If $A$ is n-generated, then $A \in \mathbf{L}_{n+2}$.

Proof. (i) Let $A \in \mathrm{S}_{\omega}$ be $n$-generated and let $F$ be a prime filter of $A$. We shall prove that the chain of prime filters containing $F$ has at most $n$-elements; whence $A \in \mathrm{S}_{n+1}$ by Proposition 1.1(ii).

Let $\Psi$ be the unique congruence on $A$ with $F$ as a congruence class. For all $a, b \in A,(a * b) \vee(b * a)=1 \in F$ and hence either $a * b \in F$ or $b * a \in F$, that is, $[a] \Psi^{F} *[b] \Psi^{F}=[1] \Psi^{F}$ or $[b] \Psi^{F} *[a] \Psi^{F}=[1] \Psi^{F}$. But a Brouwerian algebra $C$ is a chain if and only if for all $a, b \in C, a * b=1$ or $b * a=1$; thus $A / \Psi^{F}$ is a chain. Since $A / \Psi^{F}$ is generated by the images of the $n$ generators of $A$ it follows that $\left|A / \Psi^{F}\right|=n+1$. Hence the chain of all prime filters containing $F$ has at most $n$ elements by Proposition 1.1(iii).

(ii) Let $A \in \mathrm{L}_{\omega}$ be $n$-generated. Then $A$ is $(n+1)$-generated as an object of $\mathrm{S}_{\omega}$. It follows, by (i), that $A \in \mathrm{S}_{n+2}$ and hence $A \in \mathrm{L}_{n+2}$.

Our final result now follows easily; simply observe that if $B$ is an equational subclass of an equational class $\mathbf{A}$ and every $m$-generated algebra in $\mathbf{A}$ is an algebra in B, then $\mathfrak{F}_{\mathbf{A}}(m) \cong \mathfrak{F}_{\mathbf{B}}(m)$. 
THEOREM 5.7. (i) $\xi_{s_{\omega}}(m) \cong \mho_{s_{m+1}}(m)$.

(ii) $\mathfrak{F}_{\mathbf{L}_{\boldsymbol{\omega}}}(m) \cong \mathfrak{F}_{\mathbf{L}_{m+2}}(m) \cong \mathfrak{F}_{\mathbf{L}_{m+2}}(m)$.

(iii) If $n \geqslant m+1$, then $\left.\mho_{s_{n}}(m) \cong\right\}_{s_{m+1}}(m)$.

(iv) If $n \geqslant m+2$, then $\mho_{\mathbf{L}_{n}}(m) \cong \mathfrak{F}_{\mathbf{L}_{m+2}}(m)$.

(v) $\mathfrak{F}_{s_{\omega}}(m) \cong \prod_{k=0}^{m-1}\left[{ }_{0}\left(F_{s_{\omega}}(k)\right)\right]{ }^{\left(\begin{array}{c}m \\ k\end{array}\right)}$.

(vi) $\mathfrak{F}_{\mathrm{L}_{\boldsymbol{\omega}}}(m) \cong \prod_{k=0}^{m}\left[{ }_{0}\left(\mathfrak{F}_{\mathrm{S}_{\omega}}(k)\right)\right]{ }^{\left(\begin{array}{c}m \\ k\end{array}\right)} \cong \mathfrak{F}_{\mathrm{S}_{\boldsymbol{\omega}}}(m) \times_{0}\left(\mathfrak{F}_{\mathrm{S}_{\boldsymbol{\omega}}}(m)\right)$.

REMARK 5.8. The finitely generated free algebras in $\mathbf{L}_{\boldsymbol{\omega}}$ were first described by A. Horn [27]. Theorem 5.3, Theorem 5.5, and Theorem 5.7 first appeared in P. Köhler [31].

\section{BIBLIOGRAPHY}

1. R. Balbes and G. Grătzer, Injective and projective Stone algebras, Duke Math. J. 38 (1971), 339-347. MR 43 \#378.

2. R. Balbes and A. Horn, Stone lattices, Duke Math. J. 37 (1970), 537-546. MR 43 \#181.

3. - Injective and projective Heyting algebras, Trans. Amer. Math. Soc. 148 (1970), 549-559. MR 41 \#1607.

4. B. Banaschewski, injectivity and essential extensions in equational classes of algebra, Proc. Conf. on Universal Algebra (Queen's Univ., Kingston, Ont., 1969), Queen's Univ., Kingston, Ont., 1970, pp. 131-147. MR 41 \#354.

5. R. Beazer, Post-like algebras and injective Stone algebras (preprint).

6. G. Birkhoff, Lattice theory, 3rd ed., Amer. Math. Soc. Colloq. Publ., vol. 25, Amer. Math. Soc., Providence, R.I., 1967. MR 37 \#2638.

7. W. H. Cornish, Normal lattices, J. Austral. Math. Soc. 14 (1972), 200-215. MR 47 \#1703.

8. B. A. Davey, Some annihilator conditions on distributive lattices, Algebra Universalis 4 (1974), 316-322.

9.

10. A. Day, Injectivity in equational classes of algebras, Canad. J. Math. 24 (1972), 209-220. MR 45 \#145.

11. - Varieties of Heyting algebras. I (preprint).

12. - Varieties of Heyting algebras. II (preprint).

13. J. Dugundji, Topology, Allyn and Bacon, Boston, Mass., 1966. MR 33 \#1824.

14. G. Epstein, The lattice theory of Post algebras, Trans. Amer. Math. Soc. 95 (1960), 300-317. MR 22 \#3701.

15. G. A. Fraser and A. Horn, Congruence relations in direct products, Proc. Amer. Math. Soc. 26 (1970), 390-394. MR 42 \#169.

16. A. M. Gleason, Projective topological spaces, Illinois J. Math. 2 (1958), 482-489. MR 22 \#12509. \#1320.

17. G. Grätzer, Universal algebra, Van Nostrand, Princeton, N.J., 1968. MR 40

18. - Lattice theory. First concepts and distributive lattices, Freeman, San Francisco, Calif., 1971. MR 48 \#184.

19. G. Grätzer and H. Lakser, Some applications of free distributive products, Notices Amer. Math. Soc. 16 (1969), 405. Abstract \#69T-A27. 
20. G. Grätzer and H. Lakser, The structure of pseudocomplemented distributive lattices. II. Congruence extension and amalgamation, Trans. Amer. Math. Soc. 156 (1971), 343-358. MR 43 \#124.

21. The structure of pseudocomplemented distributive lattices. III. Injectives and absolute subretracts, Trans. Amer. Math. Soc. 169 (1972), 475-487. MR 46 \#8926.

22. G. Grätzer and E. T. Schmidt, On a problem of $M$. H. Stone, Acta Math. Akad. Sci. Hungar. 8 (1957), 455-460. MR 19, 1154.

23. P. R. Halmos, Lectures on Boolean algebras, Van Nostrand Studies, no. 1, Van Nostrand, Princeton, N.J., 1963. MR 29 \#4713.

24. T. Hecht and T. Katrinák, Equational classes of relative Stone algebras, Notre Dame J. Formal Logic 13 (1972), 248-254. MR 45 \#5039.

25. K. H. Hofmann, M. Mislove and A. Stralka, The Pontrjagin duality of compact 0-dimensional semilattices and its applications, Lecture Notes in Math., vol. 396, SpringerVerlag, Berlin and New York, 1974.

26. A. Horn, Logic with truth values in a linearly ordered Heyting algebra, J. Symbolic Logic 34 (1969), 395-408. MR 40 \#7089. \#7099.

27. — Free L-algebras, J. Symbolic Logic 34 (1969), 475-480. MR 40

28. B. Jónsson, Algebras whose congruence lattices are distributive, Math. Scand. 21 (1967), 110-121 MR 38 \#5689.

29. T. Katriňák, Remarks on W. C. Nemitz's paper "Semi-Boolean lattices", Notre Dame J. Formal Logic 11 (1970), 425-430. MR 45 \#40.

30. T. Katriňák and A. Mitschke, Stonesche Verbände der Ordnung $n$ und Postalgebren, Math. Ann. 199 (1972), 13-30. MR 47 \#8379.

31. P. Köhler, Freie endlich erzeugte Heyting-Algebren, Diplomarbeit, Math. Institut Giessen, 1973.

32. , Freie S-Algebren (preprint).

33. - S-Algebren mit endlichem Zentrum (preprint).

34. H. Lakser, Injective hulls of Stone algebras, Proc. Amer. Math. Soc. 24 (1970), 524-529. MR 41 \#119.

35. - The structure of pseudocomplemented distributive lattices. I. Subdirect decomposition, Trans. Amer. Math. Soc. 156 (1971), 334-342. MR 43 \#123.

36. K. B. Lee, Equational classes of distributive pseudo-complemented lattices,

Canad. J. Math. 22 (1970), 881-891. MR 42 \#151.

37. S. Mac Lane, Categories for the working mathematician, Springer-Verlag, Berlin and New York, 1971.

38. M. Mandelker, Relative annihilators in lattices, Duke Math. J. 37 (1970), 377-

386. MR 41 \#1606.

39. W. C. Nemitz, Implicative semilattices, Trans. Amer. Math. Soc. 117 (1965),

128-142. MR 31 \#1212.

40. H. A. Priestley, Representations of distributive lattices by means of ordered

Stone spaces, Bull. London Math. Soc. 2 (1970), 186-190. MR 42 \#153.

41. - Ordered topological spaces and the representation of distributive lat-

tices, Proc. London Math. Soc. Ser. (3) 24 (1972), 507-530. MR 46 \#109.

42. - Stone lattices: A topological approach, Fund Math. 84 (1974), 127143.

43. H. Rasiowa and R. Sikorski, The mathematics of metamathematics, Monografie

Mat., Tom 41, PWN, Warsaw, 1963. MR 29 \#1149.

44. T. P. Speed, Two congruences on distributive lattices. I. Prime ideals, Bull. Soc.

Roy. Sci. Liège 38 (1969), 610-628. MR 42 \#154.

45. M. H. Stone, The theory of representations for Boolean algebras, Trans. Amer. Math. Soc. 40 (1936), 37-111.

46. A. Urquhart, Free distributive pseudo-complemented lattices, Algebra Universalis 3 (1973), 13-15. 
47. A. Urquhart, Free Heyting algebras, Algebra Universalis 3 (1973), 94-97.

48. J. Varlet, On the characterization of Stone lattices, Acta Sci. Math. (Szeged) 27 (1966), 81-84. MR 33 \#2580.

DEPARTMENT OF MATHEMATICS, UNIVERSITY OF MANITOBA, WINNIPEG, MANITOBA, CANADA R3T 2N2

Current address: Department of Mathematics, LaTrobe University, Bundoora, Victoria, Australia 3083 